\title{
General structure of gauge boson propagator and its spectra in a hot magnetized medium
}

\author{
Bithika Karmakar $^{1, \mathrm{a}}$, Aritra Bandyopadhyay ${ }^{2, \mathrm{~b}}$, Najmul Haque ${ }^{3,4, \mathrm{c}}$, Munshi G. Mustafa ${ }^{1, \mathrm{~d}}$ \\ ${ }^{1}$ Theory Division, Saha Institute of Nuclear Physics, HBNI, 1/AF, Bidhannagar, Kolkata 700064, India \\ 2 Departamento de Física, Universidade Federal de Santa Maria, Santa Maria, RS 97105-900, Brazil \\ ${ }^{3}$ School of Physical Sciences, National Institute of Science Education and Research, HBNI, Khurda, Jatni 752050, India \\ ${ }^{4}$ Institut für Theoretische Physik, Justus-Liebig-Universität Giessen, 35392 Giessen, Germany
}

Received: 24 January 2019 / Accepted: 18 July 2019 / Published online: 7 August 2019

(C) The Author(s) 2019

\begin{abstract}
Based on transversality condition of gauge boson self-energy we have systematically constructed the general structure of the gauge boson two-point functions using four linearly independent basis tensors in presence of a nontrivial background i.e. hot magnetized material medium. The hard thermal loop approximation has been used for the heat bath to compute various form factors associated with the gauge boson's two point functions both in strong and weak field approximation. We have also analyzed the dispersion of a gauge boson (e.g., gluon) using the effective propagator both in strong and weak magnetic field approximation. The formalism is also applicable to QED. The presence of only thermal background leads to a longitudinal (plasmon) mode and a two fold degenerate transverse mode. In presence of a hot magnetized background medium the degeneracy of the two transverse modes is lifted and one gets three quasiparticle modes. In weak field approximation one gets two transverse modes and one plasmon mode. On the other hand, in strong field approximation also one gets the three modes in Lowest Landau Level. The general structure of two-point function may be useful for computing the thermo-magnetic correction of various quantities associated with a gauge boson.
\end{abstract}

\section{Introduction}

The propagation of vector gauge bosons in a material medium in presence of a magnetic field produces many interesting observational effects. As for example the photons with different polarizations have different dispersion properties which

\footnotetext{
a e-mail: bithika.karmakar@saha.ac.in

b e-mail: aritrabanerjee.444@gmail.com

c e-mail: nhaque@niser.ac.in

de-mail: munshigolam.mustafa@saha.ac.in
}

lead to the Faraday rotation. This has also been observed for various astrophysical objects [1-4] and in the millisecond pulsations of solar radio emission [5]. In view of the theoretical perspective the general feature is associated with the propagation of a photon in an externally magnetized material medium. The subject of the propagation of photons in magnetized plasmas has been studied in large extent and also covered in standard electromagnetic theory $[6,7]$ and plasma physics $[8,9]$ books. However, in most cases it was assumed that the medium consists of non-relativistic and nondegenerate electrons and nucleons. This suggests a modification of theoretical tools in which a general formalism based on quantum field theory proves to be helpful [10]. A quantum field theoretical formalism to calculate Faraday rotation in different kinds of media (hot magnetized one) have been done in Refs. [11,12]. Also high-intensity laser fields are used to create ultrarelativistic electron-positron plasmas which play an important role in various astrophysical situations. Some properties of such plasma are studied using QED at finite temperature $[13,14]$.

In the regime of Quantum Chromo Dynamics (QCD), nuclear matter dissolves into a thermalized color deconfined state Quark Gluon Plasma (QGP) under extreme conditions such as very high temperature and/or density. To probe different characteristics of this novel state, various high energy Heavy-Ion-Collisions (HIC) experiments are under way, e.g., RHIC@BNL, LHC@CERN and upcoming FAIR@GSI. Depending on the impact parameter of the collision, a relativistic HIC can be central or non-central. In recent years the non-central HIC is getting more and more attention in the heavy-ion community because of some distinct features which appear due to the non-centrality of the collision. One of those is the prospect of producing a very strong magnetic field in the direction perpendicular to the reaction plane due to the relatively higher rapidity of the spectator particles 
that are not participating in the collisions. Presently immense activities are in progress to study the properties of strongly interacting matter in presence of an external magnetic field, resulting in the emergence of several novel phenomena [1532]. This suggests that there is clearly an increasing demand to study the effects of intense background magnetic fields on various aspects and observables of non-central heavy-ion collisions. Also experimental evidences of photon anisotropy, provided by the PHENIX Collaboration [33], have posed a challenge for existing theoretical models. This kind of current experimental evidences have prompted that a modification of the present theoretical tools are much needed by considering the effects of intense background magnetic field on various aspects and observables of non-central HIC. In a field theoretic calculation $n$-point functions are the basic quantities to compute the various observables of a system. With this perspective in very recent works, based on various symmetries of the system for a nontrivial medium like a hot magnetized one, the general structure of fermionic 2- and 3 -point function [34], and 4-point function [35] were computed. Also the spectral representation of two point function [34] were obtained for such system. In this paper we consider gluon that propagates in a hot magnetized QCD plasma for which we aim at the general structure of the gauge boson self-energy, the effective propagator and its dispersion property. This formalism is also applicable to QED system. The general propagators for fermion obtained in Ref. [34] and for the gauge boson obtained here have already been used to compute the quark-gluon free energy for a hot magnetized deconfined QCD system in Ref. [36].

This paper has been organized as follows: in Sect. 2 the general structure of a gauge boson self-energy in a hot magnetized medium is discussed progressively. It includes two parts: a brief review of the general structure in presence of only thermal medium in Sect. 2.1 and then a generalization of it to a hot magnetized medium in Sect. 2.2. In Sect. 3 we discuss the general structure for the gauge boson propagator using the results of Sect. 2. Section 4 begins with the domain of applicability depending upon the scales (mass, temperature and the magnetic field strength) associated with the system. In Sects. 4.1 and 4.2 we elaborately compute the various form factors, Debye screening mass, dispersion relations within strong and weak field approximation, respectively. Finally, we conclude in Sect. 5.

\section{General structure of a gauge boson self-energy}

In this section we first briefly review the formalism of the general structure for a gauge boson self-energy by considering only thermal bath without the presence of any magnetic field in Sect. 2.1 and it will then be followed by a formalism for a magnetized hot medium in Sect. 2.2.

\subsection{Finite temperature and zero magnetic field case}

We begin with the general structure of the gauge boson selfenergy in vacuum, given as

$\Pi^{\mu \nu}(P)=V^{\mu \nu} \Pi\left(P^{2}\right)$,

where the form factor $\Pi\left(P^{2}\right)$ is Lorentz invariant and depends only on the four scalar $P^{2}$. The vacuum projection operator is

$V^{\mu \nu}=g^{\mu \nu}-\frac{P^{\mu} P^{\nu}}{P^{2}}$,

with the metric $g^{\mu \nu} \equiv(1,-1,-1,-1)$ and $P^{\mu} \equiv\left(p_{0}, \boldsymbol{p}\right)=$ $\left(p^{0}, p^{1}, p^{2}, p^{3}\right)$. The self-energy satisfies the gauge invariance through the transversality condition

$P_{\mu} \Pi^{\mu \nu}(P)=0$,

and it is also symmetric

$\Pi^{\mu \nu}(P)=\Pi^{v \mu}(P)$.

The conditions in Eqs. (3) and (4) are sufficient to obtain ten components of $\Pi^{\mu \nu}$.

The presence of finite temperature $(\beta=1 / T)$ or heat bath breaks the Lorentz (boost) invariance of the system. In finite temperature one accumulates four-vectors and tensors to form a general covariant structure of the gauge boson self-energy. Those are $P^{\mu}, g^{\mu \nu}$ from vacuum and the fourvelocity $u^{\mu}$ of the heat bath, discreetly introduced because of the medium. With these one can form four symmetric basis tensors, namely $P^{\mu} P^{v}, P^{\mu} u^{v}+u^{\mu} P^{v}, u^{\mu} u^{v}$ and $g^{\mu \nu}$. These four tensors can be reduced to two independent mutually orthogonal projection tensors by virtue of the constraints provided by the transversality condition in Eq. (3). One uses them to construct manifestly Lorentz-invariant structure of the gauge boson self-energy and propagator at finite temperature which have been discussed in the literature in details [37-39]. Nevertheless, we briefly discuss some of the essential points that would be very useful in constructing those general structures for a magnetized hot medium.

We now begin by defining Lorentz scalars, vectors and tensors that characterize the heat bath or hot medium in a local rest frame:

$$
\begin{aligned}
u^{\mu} & =(1,0,0,0), \\
P^{\mu} u_{\mu} & =P \cdot u=p_{0}, \\
\tilde{P}^{\mu} & =P^{\mu}-(P \cdot u) u^{\mu}=P^{\mu}-p_{0} u^{\mu}, \\
\tilde{g}^{\mu \nu} & =g^{\mu \nu}-u^{\mu} u^{v} \\
\tilde{P}^{2} & =\tilde{P}^{\mu} \tilde{P}_{\mu}=P^{2}-p_{0}^{2}=-p^{2},
\end{aligned}
$$

where $p=|p|$. We note here that one can only construct two independent Lorentz scalars as given in Eqs. (5a) and 
(5d). One can further redefine four vector $u^{\mu}$ by projecting the vacuum projection tensor upon it as

$\bar{u}^{\mu}=V^{\mu v} u_{v}=u^{\mu}-\frac{(P \cdot u) P^{\mu}}{P^{2}}=u^{\mu}-\frac{p_{0} P^{\mu}}{P^{2}}$.

which is orthogonal to $P^{\mu}$. Now one can construct two independent and mutually transverse second rank projection tensors in terms of those redefined set of four-vectors and tensor as

$$
\begin{aligned}
& A^{\mu \nu}=\tilde{g}^{\mu \nu}-\frac{\tilde{P}^{\mu} \tilde{P}^{v}}{\tilde{P}^{2},} \\
& B^{\mu \nu}=\frac{1}{\bar{u}^{2}} \bar{u}^{\mu} \bar{u}^{\nu} .
\end{aligned}
$$

Moreover, sum of these two projection operators lead to the well known vacuum projection tensor $V^{\mu \nu}$ as

$A^{\mu \nu}+B^{\mu \nu}=g^{\mu \nu}-\frac{P^{\mu} P^{\nu}}{P^{2}}=V^{\mu \nu}$.

So, the general (manifestly) covariant form of the self-energy tensor can be written as

$\Pi^{\mu v}=\Pi_{T} A^{\mu v}+\Pi_{L} B^{\mu v}$,

where $\Pi_{L}$ and $\Pi_{T}$ are, respectively, the longitudinal and transverse form factors. Eventually one can obtain these two form factors as

$$
\begin{aligned}
& \Pi_{L}=-\frac{P^{2}}{p^{2}} \Pi_{00}, \\
& \Pi_{T}=\frac{1}{D-2}\left(\Pi_{\mu}^{\mu}-\Pi_{L}\right),
\end{aligned}
$$

where $D$ is the space-time dimension of a given theory. The above Lorentz-invariant form factors would depend on the two independent Lorentz scalars $p_{0}$ and $p=\sqrt{p_{0}^{2}-P^{2}}$ as defined, respectively, in Eqs. (5a) and (5d) besides the temperature $T=1 / \beta$.

\subsection{Finite temperature and finite magnetic field case}

The finite temperature breaks the Lorentz (boost) symmetry whereas the presence of magnetic field breaks the rotational symmetry in the system. In presence of both finite temperature $(\beta=1 / T)$ and finite magnetic field $B$, the four-vectors and tensors available to form the general covariant structure of the gauge boson self-energy are $P^{\mu}, g^{\mu \nu}$, the electromagnetic field tensor $F^{\mu \nu}$ and it's dual $\tilde{F}^{\mu \nu}$, and the four velocity of the heat bath, $u^{\mu}$. As seen in Sect. 2.1 at finite $T$ the heat bath introduces a preferred direction that breaks the boost invariance. On the other hand, the presence of the magnetic field breaks the rotational symmetry in the system because it introduces an anisotropy in space. For hot magnetized system, one can define a new four vector $n^{\mu}$ which is associated with the electromagnetic field tensor $F^{\mu \nu}$. We define the electromagnetic field tensor as

$F^{\mu \nu}=\left(\begin{array}{cccc}0 & 0 & 0 & 0 \\ 0 & 0 & -B & 0 \\ 0 & B & 0 & 0 \\ 0 & 0 & 0 & 0\end{array}\right)$.

In the rest frame of the heat bath, i.e., $u^{\mu}=(1,0,0,0), n^{\mu}$ can be defined uniquely as projection of $F^{\mu v}$ along $u^{\mu}$,

$n_{\mu} \equiv \frac{1}{2 B} \epsilon_{\mu \nu \rho \lambda} u^{\nu} F^{\rho \lambda}=\frac{1}{B} u^{\nu} \tilde{F}_{\mu \nu}=(0,0,0,1)$,

which is in the $z$-direction. This also establishes a connection between the heat bath and the magnetic field.

Now for a hot magnetized case one has Lorentz vectors, $P^{\mu}, u^{\mu}$ and $n^{\mu}$ along with metric tensor $g^{\mu \nu}$, from which one can form seven symmetric basis tensors, namely $P^{\mu} P^{\nu}, P^{\mu} n^{\nu}+n^{\mu} P^{\nu}, n^{\mu} n^{\nu}, P^{\mu} u^{\nu}+u^{\mu} P^{\nu}, u^{\mu} u^{\nu}, u^{\mu} n^{\nu}+$ $n^{\mu} u^{\nu}$ and $g^{\mu \nu}$. These seven tensors reduce to four because of constraints provided by the gauge invariance condition in Eq. (3). Below we obtain the four basis tensors. ${ }^{1}$

We first form the transverse four momentum and the transverse metric tensor as

$$
\begin{aligned}
P_{\perp}^{\mu} & =P^{\mu}-(P \cdot u) u^{\mu}+(P \cdot n) n^{\mu} \\
& =P^{\mu}-p_{0} u^{\mu}+p^{3} n^{\mu}=P^{\mu}-P_{\|}^{\mu}, \\
g_{\perp}^{\mu \nu} & =g^{\mu \nu}-u^{\mu} u^{\nu}+n^{\mu} n^{\nu}=g^{\mu \nu}-g_{\|}^{\mu \nu},
\end{aligned}
$$

where

$$
\begin{aligned}
P_{\|}^{\mu} & =p_{0} u^{\mu}-p^{3} n^{\mu}, \\
P_{\|}^{2} & =P_{\|}^{\mu} P_{\mu}^{\|}=p_{0}^{2}-p_{3}^{2}, \\
g_{\|}^{\mu \nu} & =u^{\mu} u^{v}-n^{\mu} n^{v}, \\
P_{\perp}^{\mu} P_{\mu}^{\perp} & =P_{\perp}^{2}=P^{2}-p_{0}^{2}+p_{3}^{2}=P^{2}-P_{\|}^{2}=-p_{\perp}^{2},
\end{aligned}
$$

where $P^{2}=P_{\|}^{2}+P_{\perp}^{2}=P_{\|}^{2}-p_{\perp}^{2}, P_{\|}^{2}=p_{0}^{2}-p_{3}^{2}$ and $p_{\perp}^{2}=p_{1}^{2}+p_{2}^{2}$. We further note that the three independent Lorentz scalars are $p_{0}, p^{3}=P \cdot n$ and $P_{\perp}^{2}$.

We take $B^{\mu v}$ in Eq. (7b) as one of projection tensors in hot magnetized system. Now $A^{\mu v} A_{\mu \nu}=2$ indicates that it is a combination of two mutually orthogonal projection tensors, which yields two degenerate transverse modes for gauge boson in heat bath. Projection of $A^{\mu v}$ along magnetic field direction $n^{\mu}$ is $\bar{n}^{\mu}=A^{\mu v} n_{v}$. So we can construct another second rank tensor orthogonal to both $P^{\mu}$ and $B^{\mu \nu}$ as,

$Q^{\mu \nu}=\frac{\bar{n}^{\mu} \bar{n}^{\nu}}{\bar{n}^{2}}$.

\footnotetext{
${ }^{1}$ We note here that a set of four different basis tensors were used in Refs. [40-42].
} 
We, now, construct the third projection tensor $R^{\mu \nu}$, with a constraint such that the sum of $R^{\mu \nu}, B^{\mu \nu}$ and $Q^{\mu \nu}$ gives the vacuum projection operator $V^{\mu \nu}$ as

$$
\begin{aligned}
R^{\mu \nu} & =V^{\mu \nu}-B^{\mu \nu}-Q^{\mu \nu}=A^{\mu \nu}-Q^{\mu \nu} \\
& =g_{\perp}^{\mu \nu}-\frac{P_{\perp}^{\mu} P_{\perp}^{v}}{P_{\perp}^{2}} .
\end{aligned}
$$

It can be checked easily that all the projection tensors satisfy the following properties,

$$
\begin{aligned}
P_{\mu} Z^{\mu \nu} & =0, \\
Z^{\mu \lambda} Z_{\lambda}^{v} & =Z^{\mu \nu}, \\
Z^{\mu \nu} Z_{\mu \nu} & =1 .
\end{aligned}
$$

where $Z=B, R, Q$. The three projection tensors are orthogonal to each other:

$Z^{\mu v} Y_{\mu \nu}=0$,

where $Z \neq Y$ and $Y=B, R, Q$.

Now we construct the fourth tensor as

$N^{\mu \nu}=\frac{\bar{u}^{\mu} \bar{n}^{\nu}+\bar{u}^{\nu} \bar{n}^{\mu}}{\sqrt{\bar{u}^{2}} \sqrt{\bar{n}^{2}}}$,

which satisfies the following properties

$$
\begin{aligned}
N^{\mu \rho} N_{\rho v} & =B_{v}^{\mu}+Q_{v}^{\mu}, \\
B^{\mu \rho} N_{\rho v}+N^{\mu \rho} B_{\rho v} & =N_{v}^{\mu}, \\
Q^{\mu \rho} N_{\rho v}+N^{\mu \rho} Q_{\rho v} & =N_{v}^{\mu}, \\
R^{\mu \rho} N_{\rho v} & =N^{\mu \rho} R_{\rho v}=0 .
\end{aligned}
$$

Now, one can write a general covariant structure of gauge boson self-energy as

$\Pi^{\mu \nu}=b B^{\mu \nu}+c R^{\mu \nu}+d Q^{\mu \nu}+a N^{\mu \nu}$,

where $b, c, d$ and $a$ are four Lorentz-invariant form factors associated with the four basis tensors. Note that Eq. (23) can also be expressed as

$\Pi^{\mu \nu}=b B^{\mu \nu}+c A^{\mu \nu}+(d-c) Q^{\mu \nu}+a N^{\mu \nu}$

This particular decomposition of the self-energy in terms of four tensor basis is exactly same that has been used in Refs. $[43,44]$ which, however were then applied for different perspectives.

The (00) components of the constituent tensors are given by

$$
\begin{aligned}
B_{00} & =\bar{u}^{2}, \\
R_{00} & =0, \\
Q_{00} & =0, \\
N_{00} & =0, \\
\Pi_{00} & =b B_{00}=b \bar{u}^{2} .
\end{aligned}
$$

Using these information, we obtain the form factors as

$b=B^{\mu v} \Pi_{\mu \nu}$,

$c=R^{\mu v} \Pi_{\mu \nu}$,

$d=Q^{\mu \nu} \Pi_{\mu \nu}$,

$a=\frac{1}{2} N^{\mu v} \Pi_{\mu \nu}$.

In absence of the magnetic field by comparing with the known general form of finite temperature self-energy in Eq. (9), as

$\Pi_{T} A_{\mu \nu}+\Pi_{L} B_{\mu \nu}=b_{0} B_{\mu \nu}+c_{0} R_{\mu \nu}+d_{0} Q_{\mu \nu}+a_{0} N_{\mu \nu}$,

one can write

$b_{0}=\Pi_{L}$,

$c_{0}=d_{0}=\Pi_{T}$,

$a_{0}=0$

where we used the fact that $R_{\mu \nu}+Q_{\mu \nu}=A_{\mu \nu}$.

\section{General form of gauge boson propagator in a hot magnetized medium}

In covariant gauge the inverse of the gauge boson propagator in vacuum reads as

$\left(\mathcal{D}^{0}\right)_{u v}^{-1}=P^{2} g_{\mu \nu}-\frac{\xi-1}{\xi} P_{\mu} P_{\nu}$,

where $\xi$ is the gauge parameter. From Eq. (16) one can write

$P_{\mu} P_{\nu}=P^{2}\left[g_{\mu \nu}-\left(B_{\mu \nu}+R_{\mu \nu}+Q_{\mu \nu}\right)\right]$.

and using in Eq. (29), we get

$\left(\mathcal{D}^{0}\right)_{u v}^{-1}=\frac{P^{2}}{\xi} g_{\mu \nu}+P^{2} \frac{\xi-1}{\xi}\left(B_{\mu v}+R_{\mu \nu}+Q_{\mu \nu}\right)$.

The inverse of the general gauge boson propagator following Dyson-Schwinger equation reads as

$\mathcal{D}_{u v}^{-1}=\left(\mathcal{D}^{0}\right)_{u v}^{-1}-\Pi_{\mu v}$.

From Eqs. (31) and (23) we can now readily get

$$
\begin{aligned}
\mathcal{D}_{u v}^{-1}= & \frac{P^{2}}{\xi} g_{\mu \nu}+\left(P_{m}^{2}-b\right) B_{\mu \nu}+\left(P_{m}^{2}-c\right) R_{\mu \nu} \\
& +\left(P_{m}^{2}-d\right) Q_{\mu \nu}-a N_{\mu \nu},
\end{aligned}
$$

where

$P_{m}^{2}=P^{2} \frac{\xi-1}{\xi}$.

The inverse of Eq. (33) can be written as

$\mathcal{D}_{\mu \rho}=\alpha P_{\mu} P_{\rho}+\beta B_{\mu \rho}+\gamma R_{\mu \rho}+\delta Q_{\mu \rho}+\sigma N_{\mu \rho}$. 
along with

$$
\begin{aligned}
g_{\mu}^{v}= & \mathcal{D}_{\mu \rho}\left(\mathcal{D}^{\rho v}\right)^{-1} \\
= & \alpha \frac{P^{2}}{\xi} P_{\mu} P^{v}+\left[\frac{\beta P^{2}}{\xi}+\beta\left(P_{m}^{2}-b\right)-\sigma a\right] B_{\mu}^{v} \\
& +\left[\frac{\delta P^{2}}{\xi}+\delta\left(P_{m}^{2}-d\right)-\sigma a\right] Q_{\mu}^{v} \\
& +\left[\frac{\gamma P^{2}}{\xi}+\gamma\left(P_{m}^{2}-c\right)\right] R_{\mu}^{v} \\
& +\left[-\beta a+\sigma\left(P_{m}^{2}-d\right)+\frac{\sigma P^{2}}{\xi}\right] \frac{\bar{u}_{\mu} \bar{n}^{v}}{\sqrt{\bar{u}^{2}} \sqrt{\bar{n}^{2}}} \\
& +\left[-\delta a+\sigma\left(P_{m}^{2}-b\right)+\frac{\sigma P^{2}}{\xi}\right] \\
& \times \frac{\bar{n}_{\mu} \bar{u}^{v}}{\sqrt{\bar{u}^{2}} \sqrt{\bar{n}^{2}}} .
\end{aligned}
$$

Now using the explicit forms of $B_{\mu}^{\nu}, R_{\mu}^{v}, Q_{\mu}^{v}$ and $N_{\mu}^{v}$ and equating different coefficients from both sides yield the following conditions:

$$
\begin{aligned}
& \alpha=\frac{\xi}{P^{4}}, \\
& \frac{\beta P^{2}}{\xi}+\beta\left(P_{m}^{2}-b\right)-\sigma a=1, \\
& \frac{\delta P^{2}}{\xi}+\delta\left(P_{m}^{2}-d\right)-\sigma a=1, \\
& \frac{\gamma P^{2}}{\xi}+\gamma\left(P_{m}^{2}-c\right)=1, \\
& -\beta a+\sigma\left(P_{m}^{2}-d\right)+\frac{\sigma P^{2}}{\xi}=0, \\
& -\delta a+\sigma\left(P_{m}^{2}-b\right)+\frac{\sigma P^{2}}{\xi}=0 .
\end{aligned}
$$

Solving this we get

$$
\begin{aligned}
\alpha & =\frac{\xi}{P^{4}}, \\
\beta & =\frac{P^{2}-d}{\left(P^{2}-b\right)\left(P^{2}-d\right)-a^{2}}, \\
\gamma & =\frac{1}{P^{2}-c}, \\
\delta & =\frac{P^{2}-b}{\left(P^{2}-b\right)\left(P^{2}-d\right)-a^{2}}, \\
\sigma & =\frac{a}{\left(P^{2}-b\right)\left(P^{2}-d\right)-a^{2}} .
\end{aligned}
$$

Now the general covariant structure of the gauge boson propagator in covariant gauge can finally be obtained as

$$
\mathcal{D}_{\mu \nu}=\frac{\xi P_{\mu} P_{\nu}}{P^{4}}+\frac{\left(P^{2}-d\right) B_{\mu \nu}}{\left(P^{2}-b\right)\left(P^{2}-d\right)-a^{2}}+\frac{R_{\mu \nu}}{P^{2}-c}
$$

$$
\begin{aligned}
& +\frac{\left(P^{2}-b\right) Q_{\mu \nu}}{\left(P^{2}-b\right)\left(P^{2}-d\right)-a^{2}} \\
& +\frac{a N_{\mu \nu}}{\left(P^{2}-b\right)\left(P^{2}-d\right)-a^{2}} .
\end{aligned}
$$

We recall that the breaking of boost invariance due to finite temperature leads to two modes (degenerate transverse mode and plasmino). Now, the breaking of the rotational invariance in presence of magnetic field lifts the degeneracy of the transverse modes which introduces an additional mode in the hot medium. These three dispersive modes of gauge boson can be seen from the poles of Eq. (39). The poles $\left(P^{2}-b\right)\left(P^{2}-d\right)-a^{2}=0$, lead to two dispersive modes. We call one mode $n^{+}$with energy $\omega_{n^{+}}$and the other one $n^{-}$ with energy $\omega_{n^{-}}$. The pole $P^{2}-c=0$ leads to the third dispersive mode $c$ with energy $\omega_{c}$. We will discuss about these dispersive modes in details later for both strong and weak field approximation.

When we turn off the magnetic field, the general structure of the propagator in a non-magnetized thermal bath can be obtained by putting $b_{0}=\Pi_{L}, c_{0}=d_{0}=\Pi_{T}$ and $a_{0}=0$ as

$\mathcal{D}_{\mu \rho}=\frac{\xi P_{\mu} P_{\rho}}{P^{4}}+\frac{B_{\mu \rho}}{P^{2}-\Pi_{L}}+\frac{A_{\mu \rho}}{P^{2}-\Pi_{T}}$

which agrees with the known result $[37-39,45]$.

\section{Form factors}

Before computing the various form factors associated with the general structure we note the following points:

1. The magnetic field generated during the non-central HIC is time dependent but is believed to decrease rapidly with time [20,46]. It would be extremely complicated to work with a time dependent magnetic field. Instead we work by considering a constant background magnetic field along with some limiting conditions so that the effect of magnetic field can be incorporated analytically. We note here that incorporation of magnetic field to the heat bath introduces another scale in the system. Beside the fermion mass $m_{f}$ and the temperature $T$, the additional scale is the strength of magnetic field $B$. Below we would discuss the different domains of scales:

a) Strong Field Approximation: At the time of the collision, the value of the magnetic field $B$ is estimated upto the order of $|e B| \sim 15 m_{\pi}^{2}$ (where $e$ is the electronic charge, $m_{\pi}$ is the mass of a pion), which is very high compared to the temperature $T$ and $m_{f}$ in the LHC at CERN [47]. Also in the dense sector, neutron stars (NS), or more specifically magnetars are known to possess strong enough magnetic field [48-50]. The effect of this strong enough magnetic field can be incorporated via 


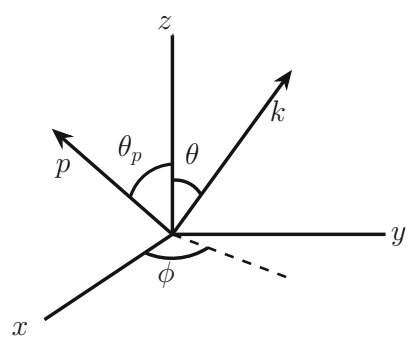

Fig. 1 Choice of reference frame for computing the various form factors associated with the general structure of gauge boson 2-point functions. The magnetic field is along $z$-direction

a simplified Lowest Landau Level (LLL) approximation in which fermions are basically confined within the LLL. In the Sect. 4.1 we will work on strong field approximation with a scale hierarchy, $m_{f}<T<\sqrt{|e B|}$, where the loop momentum $K \sim T$ within HTL approximation.

b) Weak Field Approximation: Furthermore, it is believed that the magnetic field generated in heavy-ion collisions decreases rapidly with time. This provides us a simplified situation where one can work in weak field approximation with a scale hierarchy, $\sqrt{|e B|}<m_{f}<T$ which will be discussed in details in Sect. 4.2.

2. We would consider $m_{f}=5 \mathrm{MeV}$ for two light quark flavors $u$ and $d$.

3. We choose a frame of reference as shown in Fig. 1 in which one considers the external momentum of the vector boson in $x z$ plane $^{2}$ with $0<\theta_{p}<\pi / 2$. So one can write

$P^{\mu}=\left(p_{0}, p \sin \theta_{p}, 0, p \cos \theta_{p}\right)$

and then loop momenta

$K^{\mu}=\left(k_{0}, k \sin \theta \cos \phi, k \sin \theta \sin \phi, k \cos \theta\right)$.

\subsection{Gauge boson in strongly magnetized medium}

\subsubsection{One-loop gluon self-energy}

When the external magnetic field is very strong, $e B \rightarrow \infty$, it pushes all the Landau levels $(n \geq 1)$ to infinity compared to the Lowest Landau Level (LLL) with $n=0$. For LLL approximation in the strong field limit the fermion propagator reduces to a simplified form as

\footnotetext{
${ }^{2}$ However, one can consider a general frame of reference $P_{\mu}=$ $\left(p_{0}, p_{1}, p_{2}, p_{3}\right)$ and the result would be independent of the choice of reference frame. Because $p_{\perp}$ and $p_{3}$ are not in same footing due to the anisotropy caused by the external magnetic field along $z$ direction. But there is no distinction between $p_{1}$ and $p_{2}$. So, for simplicity of the calculation, we made a particular choice for the reference frame here.
}

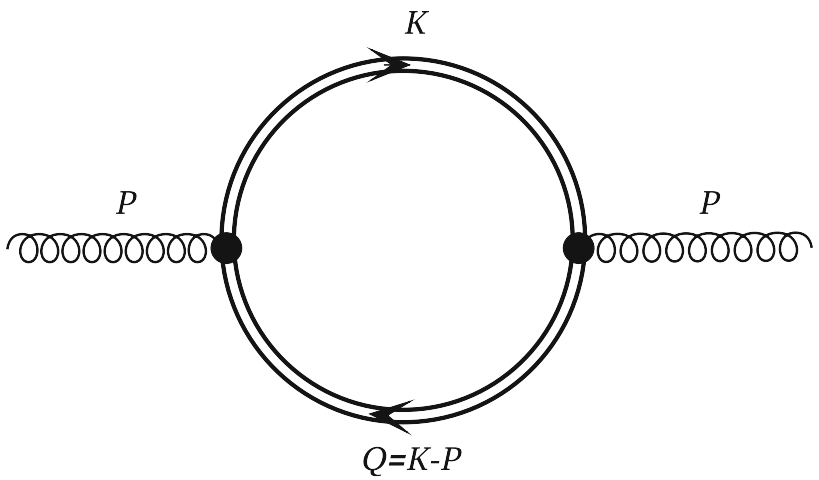

Fig. 2 Gluon polarization tensor in the limit of strong field approximation

$i S_{m}^{s}(K)=i e^{-k_{\perp}^{2} /\left|q_{f} B\right|} \frac{K_{\|}+m_{f}}{K_{\|}^{2}-m_{f}^{2}}\left(1-i \gamma_{1} \gamma_{2}\right)$,

where $K$ is the fermionic four momentum and we have used the properties of generalized Laguerre polynomial, $L_{n} \equiv L_{n}^{0}$ and $L_{-1}^{\alpha}=0$. In strong field approximation or in LLL, $e B \gg$ $k_{\perp}^{2}$, an effective dimensional reduction from $(3+1)$ to $(1+1)$ takes place.

Now in the strong field limit the self-energy (Fig. 2) can be computed as

$$
\begin{aligned}
& \Pi_{\mu \nu}^{s}(P) \\
&= \sum_{f} \frac{i g^{2}}{2} \int \frac{d^{4} K}{(2 \pi)^{4}} \operatorname{Tr}\left[\gamma_{\mu} S_{m}^{S}(K) \gamma_{\nu} S_{m}^{S}(Q)\right] \\
&= \sum_{f} \frac{i g^{2}}{2} \int \frac{d^{2} k_{\perp}}{(2 \pi)^{2}} \exp \left(\frac{-k_{\perp}^{2}-q_{\perp}^{2}}{\left|q_{f} B\right|}\right) \\
& \quad \times \int \frac{d^{2} K_{\|}}{(2 \pi)^{2}} \operatorname{Tr}\left[\gamma_{\mu} \frac{\mathbb{K}_{\|}+m_{f}}{K_{\|}^{2}-m_{f}^{2}}\left(1-i \gamma_{1} \gamma_{2}\right) \gamma_{\nu}\right. \\
&\left.\quad \times \frac{Q_{\|}+m_{f}}{Q_{\|}^{2}-m_{f}^{2}}\left(1-i \gamma_{1} \gamma_{2}\right)\right],
\end{aligned}
$$

where ' $s$ ' indicates that the quantities are to be calculated in the strong field approximation and $\mathrm{Tr}$ represents only the Dirac trace. We have suppressed the color indices for convenience. Now one can notice that the longitudinal and transverse parts are completely separated and the Gaussian integration over the transverse momenta can be done trivially, which leads to

$$
\begin{aligned}
\Pi_{\mu \nu}^{s}(P)= & \sum_{f} i e^{-p_{\perp}^{2} / 2\left|q_{f} B\right|} \frac{g^{2}\left|q_{f} B\right|}{2 \pi} \\
& \times \int \frac{d^{2} K_{\Perp}}{(2 \pi)^{2}} \frac{\mathcal{S}_{\mu \nu}^{s}}{\left(K_{\|}^{2}-m_{f}^{2}\right)\left(Q_{\|}^{2}-m_{f}^{2}\right)} \\
= & -\sum_{f} e^{-p_{\perp}^{2} / 2\left|q_{f} B\right|} \frac{g^{2}\left|q_{f} B\right|}{2 \pi} T \sum_{k_{0}}
\end{aligned}
$$




$$
\times \int \frac{d k_{3}}{2 \pi} \frac{\mathcal{S}_{\mu \nu}^{s}}{\left(K_{\|}^{2}-m_{f}^{2}\right)\left(Q_{\|}^{2}-m_{f}^{2}\right)},
$$

with the tensor structure $\mathcal{S}_{\mu \nu}^{s}$ that originates from the Dirac trace is

$\mathcal{S}_{\mu \nu}^{s}=K_{\mu}^{\|} Q_{\nu}^{\|}+Q_{\mu}^{\|} K_{\nu}^{\|}-g_{\mu \nu}^{\|}\left((K \cdot Q)_{\|}-m_{f}^{2}\right)$,

where the Lorentz indices $\mu$ and $v$ are restricted to longitudinal values because of dimensional reduction to $(1+1)$ dimension and forbids to take any transverse values. Now we use Eqs. (14a) and (14c) to rewrite $S_{\mu \nu}$ as

$$
\begin{aligned}
\mathcal{S}_{\mu \nu}^{s}= & \left(k_{0} u_{\mu}-k_{3} n_{\mu}\right)\left(q_{0} u_{v}-q_{3} n_{v}\right) \\
& +\left(q_{0} u_{\mu}-q_{3} n_{\mu}\right)\left(k_{0} u_{v}-k_{3} n_{v}\right) \\
& -\left(u_{\mu} u_{v}-n_{\mu} n_{\nu}\right)\left((k \cdot q)_{\|}-m_{f}^{2}\right) \\
= & u_{\mu} u_{v}\left(k_{0} q_{0}+k_{3} q_{3}+m_{f}^{2}\right) \\
& +n_{\mu} n_{v}\left(k_{0} q_{0}+k_{3} q_{3}-m_{f}^{2}\right) \\
& -\left(u_{\mu} n_{v}+n_{\mu} u_{v}\right)\left(k_{0} q_{3}+k_{3} q_{0}\right) .
\end{aligned}
$$

\subsubsection{Form factors and Debye mass}

First we evaluate the form factors in Eqs. (26a), (26b), (26c) and (26d) in strong field approximation as

$$
\begin{aligned}
c= & R^{\mu \nu}\left(\Pi_{\mu \nu}^{g}+\Pi_{\mu \nu}^{s}\right)=c_{Y M}+c_{s} \\
= & \frac{C_{A} g^{2} T^{2}}{3} \frac{1}{2}\left[\frac{p_{0}^{2}}{p^{2}}-\frac{P^{2}}{p^{2}} \mathcal{T}_{P}\left(p_{0}, p\right)\right] \text { where } c_{s}=0 \\
b= & B^{\mu \nu}\left(\Pi_{\mu \nu}^{g}+\Pi_{\mu \nu}^{s}\right)=b_{Y M}+\frac{u^{\mu} u^{\nu}}{\bar{u}^{2}} \Pi_{\mu \nu}^{s} \\
= & b_{Y M}+b_{s}=\frac{C_{A} g^{2} T^{2}}{3 \bar{u}^{2}}\left[1-\mathcal{T}_{P}\left(p_{0}, p\right)\right] \\
& -\sum_{f} e^{-p_{\perp}^{2} / 2\left|q_{f} B\right|} \frac{g^{2}\left|q_{f} B\right|}{2 \pi \bar{u}^{2}} T \\
& \times \sum_{k_{0}} \int \frac{d k_{3}}{2 \pi} \frac{k_{0} q_{0}+k_{3} q_{3}+m_{f}^{2}}{\left(K_{\|}^{2}-m_{f}^{2}\right)\left(Q_{\|}^{2}-m_{f}^{2}\right)} \\
d= & d_{Y M}+Q^{\mu \nu} \Pi_{\mu \nu}^{s}=d_{Y M}+d_{s} \\
= & \frac{C_{A} g^{2} T^{2}}{3} \frac{1}{2}\left[\frac{p_{0}^{2}}{p^{2}}-\frac{P^{2}}{p^{2}} \mathcal{T}_{P}\left(p_{0}, p\right)\right] \\
& +\sum_{f} e^{-p_{\perp}^{2} / 2\left|q_{f} B\right|} \frac{g^{2}\left|q_{f} B\right|}{2 \pi} \frac{p_{\perp}^{2}}{p^{2}} T \\
& \times \sum_{k_{0}} \int \frac{d k_{3}}{2 \pi} \frac{k_{0} q_{0}+k_{3} q_{3}-m_{f}^{2}}{\left(K_{\|}^{2}-m_{f}^{2}\right)\left(Q_{\|}^{2}-m_{f}^{2}\right)} \\
a= & \frac{1}{2} N^{\mu \nu}\left(\Pi_{\mu \nu}^{g}+\Pi_{\mu \nu}^{s}\right) \\
&
\end{aligned}
$$

$$
=\frac{1}{2} N^{\mu \nu} \Pi_{\mu \nu}^{s}=a_{s} \text {, where } a_{Y M}=0,
$$

where $\Pi_{\mu \nu}^{g}$ is the Yang-Mills (YM) contribution from ghost and gluon loop which remain unaffected in presence of magnetic field and can be written as

$\Pi_{\mu \nu}^{g}(P)=-\frac{N_{c} g^{2} T^{2}}{3} \int \frac{d \Omega}{2 \pi}\left(\frac{p_{0} \hat{K}_{\mu} \hat{K}_{\nu}}{\hat{K} \cdot P}-g_{\mu 0} g_{\nu 0}\right)$.

Now, combining Eq. (48b) and the Hard Thermal Loop (HTL) approximation [51] one can have

$$
\begin{aligned}
b_{s} \approx & -\sum_{f} e^{-p_{\perp}^{2} / 2\left|q_{f} B\right|} \frac{g^{2}\left|q_{f} B\right|}{2 \pi \bar{u}^{2}} T \sum_{k_{0}} \int \frac{d k_{3}}{2 \pi} \\
& \times\left[\frac{1}{\left(K_{\|}^{2}-m_{f}^{2}\right)}+\frac{2\left(k_{3}^{2}+m_{f}^{2}\right)}{\left(K_{\|}^{2}-m_{f}^{2}\right)\left(Q_{\|}^{2}-m_{f}^{2}\right)}\right] \\
= & \sum_{f} e^{-p_{\perp}^{2} / 2\left|q_{f} B\right|} \frac{g^{2}\left|q_{f} B\right|}{2 \pi \bar{u}^{2}} \int \frac{d k_{3}}{2 \pi} \\
& \times\left[-\frac{n_{F}\left(E_{k_{3}}\right)}{E_{k_{3}}}+\left\{\frac{n_{F}\left(E_{k_{3}}\right)}{E_{k_{3}}}+\frac{p_{3} k_{3}}{E_{k_{3}}} \frac{\partial n_{F}\left(E_{k_{3}}\right)}{\partial k_{3}}\right.\right. \\
& \left.\left.\times\left(\frac{p_{3} k_{3} / E_{k_{3}}}{p_{0}^{2}-p_{3}^{2}\left(k_{3} / E_{k_{3}}\right)^{2}}\right)\right\}\right] \\
= & \sum_{f} e^{-p_{\perp}^{2} / 2\left|q_{f} B\right|} \frac{g^{2}\left|q_{f} B\right|}{2 \pi \bar{u}^{2}} \int \frac{d k_{3}}{2 \pi} \frac{p_{3} k_{3}}{E_{k_{3}}} \frac{\partial n_{F}\left(E_{k_{3}}\right)}{\partial E_{k_{3}}} \\
& \times\left(\frac{p_{3} k_{3} / E_{k_{3}}}{p_{0}^{2}-p_{3}^{2}\left(k_{3} / E_{k_{3}}\right)^{2}}\right) .
\end{aligned}
$$

Using Eqs. (48b), (50) in Eq. (25e) one also can directly calculate the Debye screening mass in QCD as

$$
\begin{aligned}
\left(m_{D}^{2}\right)_{s}= & \left.\bar{u}^{2} b\right|_{p_{0}=0, p \rightarrow 0}=m_{D}^{2}+\sum_{f}\left(\delta m_{D, f}^{2}\right)_{s} \\
= & m_{D}^{2}-\sum_{f} \frac{g^{2}\left|q_{f} B\right|}{2 \pi} \int \frac{d k_{3}}{2 \pi} \frac{\partial n_{F}\left(E_{k_{3}}\right)}{\partial E_{k_{3}}} \\
= & \frac{g^{2} N_{c} T^{2}}{3}+\sum_{f} \frac{g^{2}\left|q_{f} B\right|}{2 \pi T} \\
& \times \int_{-\infty}^{\infty} \frac{d k_{3}}{2 \pi} n_{F}\left(E_{k_{3}}\right)\left(1-n_{F}\left(E_{k_{3}}\right)\right) .
\end{aligned}
$$

which reduces to the expression of QED Debye mass calculated in Refs. [52,53] without QCD factors where three distinct scales $\left(m_{f}^{2}, T^{2}\right.$ and $\left.e B\right)$ were clearly evident for massive quarks.

Now using Eq. (51) in Eq. (50) along with $E_{k_{3}} \sim k_{3}$, the form factor $b$ can be expressed in terms of $m_{D}$ as 


$$
\begin{aligned}
b= & \frac{C_{A} g^{2} T^{2}}{3 \bar{u}^{2}}\left[1-\mathcal{T}_{P}\left(p_{0}, p\right)\right] \\
& -\sum_{f} e^{-p_{\perp}^{2} / 2\left|q_{f} B\right|}\left(\frac{\delta m_{D, f}}{\bar{u}}\right)^{2} \frac{p_{3}^{2}}{p_{0}^{2}-p_{3}^{2}} .
\end{aligned}
$$

The form factor $d$ then becomes

$$
\begin{aligned}
d \approx & \frac{C_{A} g^{2} T^{2}}{3} \frac{1}{2}\left[\frac{p_{0}^{2}}{p^{2}}-\frac{P^{2}}{p^{2}} \mathcal{T}_{P}\left(p_{0}, p\right)\right] \\
& +\sum_{f} e^{-p_{\perp}^{2} / 2\left|q_{f} B\right|}\left(\frac{\delta m_{D, f}}{\bar{u}}\right)^{2} \frac{p_{3}^{2}}{p_{0}^{2}-p_{3}^{2}} .
\end{aligned}
$$

where the expression for $\left(\Pi_{\mu}^{\mu}\right)^{s}$ is given in Eq. (B2) in Appendix B.

The form factor $d_{s}$ can be calculated as

$$
\begin{aligned}
d_{s}= & Q^{\mu \nu} \Pi_{\mu \nu}^{s}, \\
\approx & -\sum_{f} i e^{-p_{\perp}^{2} / 2\left|q_{f} B\right|} \frac{g^{2}\left|q_{f} B\right|}{2 \pi} \frac{p_{\perp}^{2}}{p^{2}} \\
& \times \int \frac{d^{2} K_{\Perp}}{(2 \pi)^{2}}\left[\frac{\left(k_{0}^{2}+k_{3}^{2}-m_{f}^{2}\right)}{\left(K_{\|}^{2}-m_{f}^{2}\right)\left(Q_{\|}^{2}-m_{f}^{2}\right)}\right], n n \\
\approx & \sum_{f} e^{-p_{\perp}^{2} / 2\left|q_{f} B\right|} \delta m_{D, f}^{2} \frac{p_{\perp}^{2}}{p^{2}} \frac{p_{3}^{2}}{p_{0}^{2}-p_{3}^{2}}
\end{aligned}
$$

for $k_{3} \sim E_{k_{3}}$. Now using (55) in (48d), the form factor $d$ can be written as

$$
\begin{aligned}
d \approx & \frac{C_{A} g^{2} T^{2}}{3} \frac{1}{2}\left[\frac{p_{0}^{2}}{p^{2}}-\frac{P^{2}}{p^{2}} \mathcal{T}_{P}\left(p_{0}, p\right)\right] \\
& +\sum_{f} e^{-p_{\perp}^{2} / 2\left|q_{f} B\right|} \delta m_{D, f}^{2} \frac{p_{\perp}^{2}}{p^{2}} \frac{p_{3}^{2}}{p_{0}^{2}-p_{3}^{2}},
\end{aligned}
$$

where $p_{3}=p \cos \theta_{p}$ and $p_{\perp}=p \sin \theta_{p}$ as given in Eq. (41).

Also

$$
\begin{aligned}
2 a= & N^{\mu \nu} \Pi_{\mu \nu}^{s}=\sum_{f} i e^{-p_{\perp}^{2} / 2\left|q_{f} B\right|} \frac{g^{2}\left|q_{f} B\right|}{2 \pi \sqrt{\bar{u}^{2}} \sqrt{\bar{n}^{2}}} \\
& \times \int \frac{d^{2} K_{\|}}{(2 \pi)^{2}}\left[\frac{-2 \frac{\bar{u} \cdot n}{\bar{u}^{2}}\left(k_{0}^{2}+k_{3}^{2}+m_{f}^{2}\right)+4 k_{0} k_{3}}{\left(K_{\|}^{2}-m_{f}^{2}\right)\left(Q_{\|}^{2}-m_{f}^{2}\right)}\right] \\
= & \sum_{f} e^{-p_{\perp}^{2} / 2\left|q_{f} B\right|} \frac{g^{2}\left|q_{f} B\right|}{2 \pi \sqrt{\bar{u}^{2}} \sqrt{\bar{n}^{2}}} \\
& \times \int \frac{d k_{3}}{2 \pi}\left[-2 \frac{\bar{u} \cdot n}{\bar{u}^{2}} \frac{\partial n_{F}\left(E_{k_{3}}\right)}{\partial E_{k_{3}}} \frac{p_{3}^{2} k_{3}^{2} / E_{k_{3}}^{2}}{\left(p_{0}^{2}-p_{3}^{2} k_{3}^{2} / E_{k_{3}}^{2}\right)}\right. \\
& \left.+\frac{2 \partial n_{F}\left(E_{k_{3}}\right)}{\partial E_{k_{3}}} \frac{p_{0} p_{3} k_{3}^{2} / E_{k_{3}}^{2}}{\left(p_{0}^{2}-p_{3}^{2} k_{3}^{2} / E_{k_{3}}^{2}\right)}\right] \\
\approx & \sum_{f} 2 e^{-p_{\perp}^{2} / 2\left|q_{f} B\right|} \frac{\sqrt{\bar{n}^{2}}}{\sqrt{\bar{u}^{2}}} \delta m_{D, f}^{2} \frac{p_{0} p_{3}}{p_{0}^{2}-p_{3}^{2}},
\end{aligned}
$$

where $\bar{n}^{2}=-p_{\perp}^{2} / p^{2}=-\sin ^{2} \theta_{p}$ and $\bar{u}^{2}=-p^{2} / P^{2}$.

Also in the strong field approximation, $|e B|>T^{2}>$ $m_{f}^{2}$, one can neglect the quark mass $m_{f}$, to get an analytic expression of Debye mass as

$$
\begin{aligned}
\left(m_{D}^{2}\right)_{s}= & \frac{g^{2} N_{c} T^{2}}{3} \\
& +\sum_{f} \frac{g^{2}\left|q_{f} B\right|}{2 \pi T} \int_{-\infty}^{\infty} \frac{d k_{3}}{2 \pi} n_{F}\left(k_{3}\right)\left(1-n_{F}\left(k_{3}\right)\right) \\
= & \frac{g^{2} N_{c} T^{2}}{3}+\sum_{f} \frac{g^{2}\left|q_{f} B\right|}{4 \pi^{2}} \\
= & m_{D}^{2}+\sum_{f}\left(\delta m_{D, f}^{2}\right)_{s} \\
= & m_{D}^{2}+\left(\delta m_{D}^{2}\right)_{s},
\end{aligned}
$$

which agrees with that obtained in Ref. [53].

\subsubsection{Dispersion}

As discussed after Eq. (39), the dispersion relations for gluon in strong field approximation with LLL read as

$$
\begin{aligned}
P^{2}-c & =0, \\
\left(P^{2}-b\right)\left(P^{2}-d\right)-a^{2} & =\left(P^{2}-\omega_{n}^{+}\right)\left(P^{2}-\omega_{n}^{-}\right)=0,
\end{aligned}
$$

with

$\omega_{n^{+}}=\frac{b+d+\sqrt{(b-d)^{2}+4 a^{2}}}{2}$,

$\omega_{n^{-}}=\frac{b+d-\sqrt{(b-d)^{2}+4 a^{2}}}{2}$,

where the form factors are given, respectively, in Eqs. (48a), (52), (56) and (57).

The solutions of above three dispersion relations are named as $c$-mode, $n^{+}$-mode and $n^{-}$-mode with energies $\omega_{c}$, $\omega_{n^{+}}$and $\omega_{n^{-}}$, respectively. The dispersion plot for the three modes of gluon in strong field approximation is shown in Fig. 3 for $|e B|=20 m_{\pi}^{2}, T=0.2 \mathrm{GeV}$ and for three propagation angles $\theta_{p}=0, \pi / 4$ and $\pi / 2$. We have used both magnetic field and temperature dependent coupling constant [36] for the purpose. As found $c_{s}=0$ in Eq. (48a) which implies that the $c$-mode is unaffected by the magnetic field and propagates like HTL transverse mode irrespective of the propagation angle as shown in Fig. 3. The reason for which could be understood in the following way: in strong field approximation there is an effective dimensional reduction from $(3+1)$ to $(1+1)$ dimension in LLL. Fermions at LLL can move only along the direction of external magnetic field. The electric field corresponding to the $c$ mode is always transverse to the external magnetic field irrespective of the prop- 

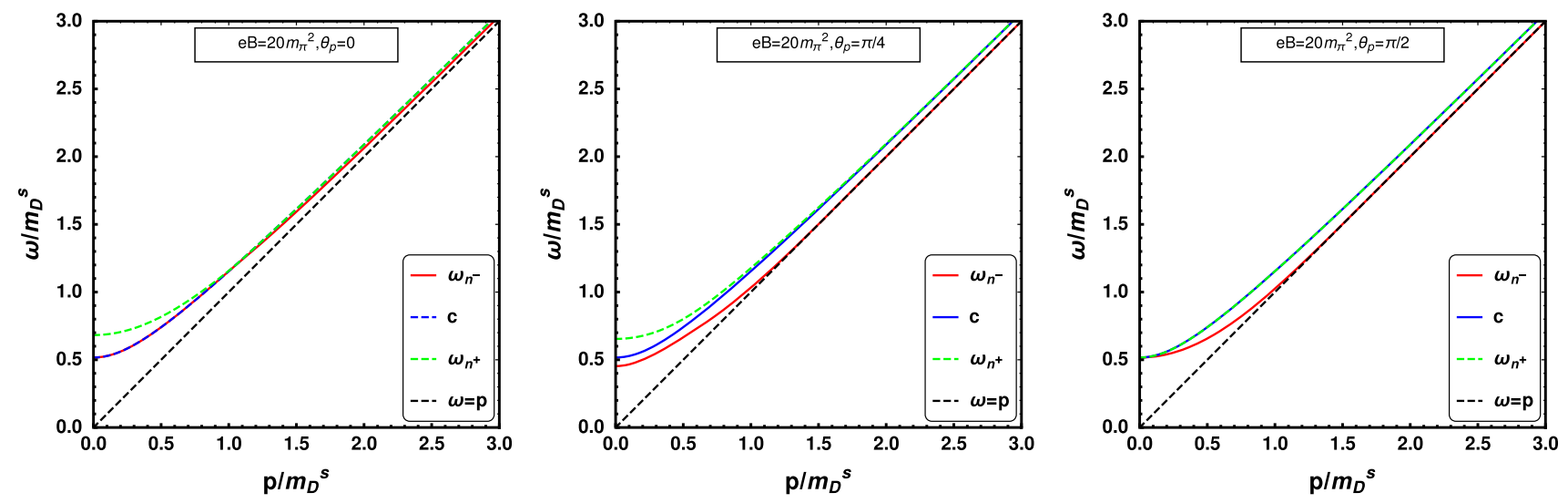

Fig. 3 The plot of dispersion of the three modes $\left(n^{-}, c\right.$ and $n^{+}$modes) of a gauge boson in strong field approximation for propagation angles $\theta_{p}=0, \pi / 4$ and $\pi / 2$ at $e B=20 m_{\pi}^{2}$ and $T=0.2 \mathrm{GeV} . \omega=p$ represents the light cone

agation angle of gluon. Thus, the fermions are not affected by the gluon excitation [40] and the quark loop contribution $\left(c_{s}\right)$ becomes zero.

Now we note that at $\theta_{p}=0$ the form factor $a=0$ as it is proportional to $\sin \theta_{p} \cos \theta_{p}$. In this case both $n^{-}$and $c$ modes are degenerate as the form factors coincide with the HTL $\Pi_{T}$ without the quark loop contribution. This is because quark loop contribution in the form factor $d$ in Eq. (56) is proportional to $\sin ^{2} \theta_{p} \cos ^{2} \theta_{p}$. This makes $n^{-}$and $c$ mode to coincide with the HTL transverse dispersive mode. This can be seen from the left panel of Fig. 3. It could also be understood in the following way: when gluon propagates along the direction of external magnetic field, i.e., $\theta_{p}=0$, the two transverse modes become rotationally symmetric about the external magnetic field and become degenerate which is shown in the left panel of Fig. 3. The electric fields corresponding to the $n^{-}$and $c$ modes are perpendicular to the external magnetic field. Thus two transverse electric fields can not excite the fermions whose movement are restricted to the direction of external magnetic field in LLL [40]. This makes the quark loop contribution zero as noted earlier. In addition to the two transverse modes $n^{-}$and $c$, there is also a longitudinal excitation $n^{+}$at $\theta_{p}=0$. At any intermediate angle of propagation, e.g, $\theta_{p}=\pi / 4$, the degeneracy of the transverse modes is lifted as shown in the middle panel of Fig. 3. Here both the transverse and longitudinal modes can excite the fermions as the corresponding electric fields are not orthogonal to the external magnetic field. As the propagation angle increases, the pole position corresponding to the $n^{-}$mode shifts from transverse channel and approaches the longitudinal channel [40]. At $\theta_{p}=\pi / 2$, the form factor $a$ in Eq. (57) and the quark contribution of the form factor $d$ in Eq. (56) also vanish because of their $\theta_{p}$ dependence. Thus, the $n^{-}$mode merges with HTL longitudinal mode whereas the $n^{+}$mode merges with $c$ mode. This is reflected in the right panel of Fig. 3.
4.2 Gauge boson in weakly magnetized hot medium

\subsubsection{One-loop gluon self-energy}

The fermion propagator in a weak magnetic field, i.e., $\sqrt{|e B|}<(K \sim T)$ and $m_{f}$, can be written up to $\mathcal{O}\left[(e B)^{2}\right]$ as

$$
\begin{aligned}
i S_{m}^{w}(K)= & i \frac{\not K+m_{f}}{K^{2}-m_{f}^{2}} \\
& +i\left(q_{f} B\right) \frac{\left(\gamma_{5}\{(K \cdot n) \not h-(K \cdot u) \not h\}+i \gamma_{1} \gamma_{2} m_{f}\right)}{\left(K^{2}-m_{f}^{2}\right)^{2}} \\
& +i 2\left(q_{f} B\right)^{2} \\
& \times\left[\frac{\{(K \cdot u) \not h-(K \cdot n) \not h\}-\not K}{\left(K^{2}-m_{f}^{2}\right)^{3}}-\frac{k_{\perp}^{2}\left(\not K+m_{f}\right)}{\left(K^{2}-m_{f}^{2}\right)^{4}}\right] \\
& +\mathcal{O}\left[(e B)^{3}\right] \\
= & S_{0}+S_{1}+S_{2}+\mathcal{O}\left[(e B)^{3}\right],
\end{aligned}
$$

where $S_{0}$ is the continuum free field propagator in absence of $B$ whereas $S_{1}$ and $S_{2}$ are, respectively, $\mathcal{O}[(e B)]$ and $\mathcal{O}\left[(e B)^{2}\right]$ correction terms in presence of $B$. The contribution to the gluon self-energy due to the quark loop can be written from the Feynman diagram Fig. 4 as

$$
\Pi_{\mu \nu}^{w, q}(P)=\sum_{f} \frac{i g^{2}}{2} \int \frac{d^{4} K}{(2 \pi)^{4}} \operatorname{Tr}\left[\gamma_{\mu} S_{m}^{w}(K) \gamma_{\nu} S_{m}^{w}(Q)\right]
$$

We have suppressed the color indices here also for convenience. Using Eq. (61) the self-energy in weak field approximation upto an $\mathcal{O}\left[(e B)^{2}\right]$ and also adding pure YM contribution, total gluon self-energy in weak field approximation can be decomposed as 


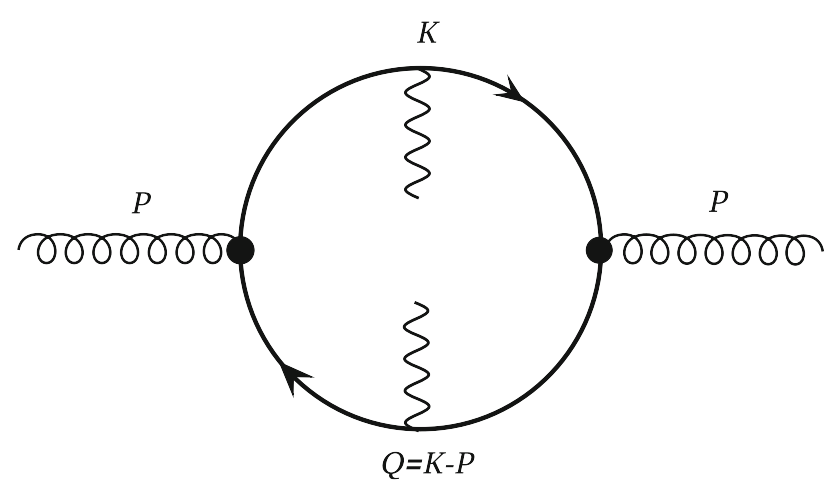

Fig. 4 The order of $(e B)^{2}$ correction to the gluon polarization tensor $\left(\delta \Pi_{\mu \nu}^{a}\right)$ in weak field approximation

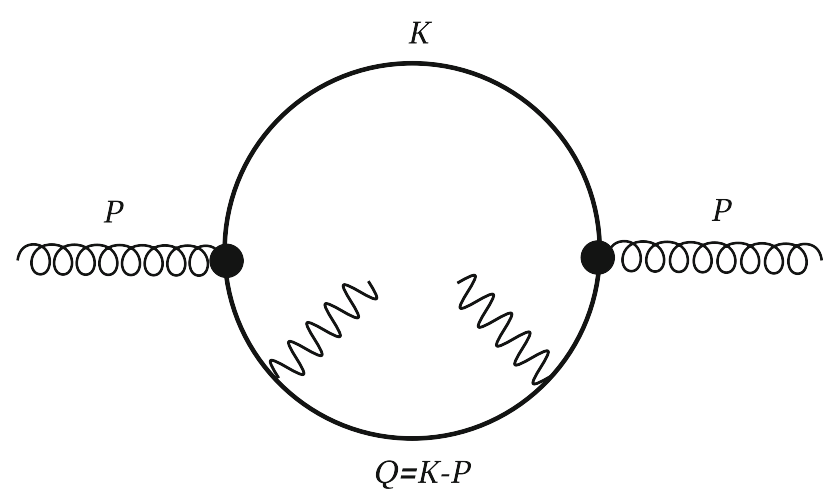

Fig. 5 The order of $(e B)^{2}$ correction to the gluon polarization tensor $\left(\delta \Pi_{\mu \nu}^{b}\right)$ in weak field approximation

$$
\begin{aligned}
\Pi_{\mu \nu}^{w}(P)= & \Pi_{\mu \nu}^{g}(P)+\Pi_{\mu \nu}^{0}(P)+\delta \Pi_{\mu \nu}^{a}(P) \\
& +2 \delta \Pi_{\mu \nu}^{b}(P)+\mathcal{O}\left[(e B)^{3}\right],
\end{aligned}
$$

where the first term $\Pi_{\mu \nu}^{g}$ is the YM contribution which is given in Eq. (49). The last three terms in Eq. (63) appear from the expansion of quark loop contribution to the gluon selfenergy. The term $\Pi_{\mu \nu}^{0}$, containing two $S_{0}$, is the leading order perturbative term in absence of $B$ whereas the remaining two terms are $(e B)^{2}$ order corrections as shown in Figs. 4 and 5. However, we note that $\mathcal{O}[(e B)]$ vanishes according to Furry's theorem since the expectation value of any odd number of electromagnetic currents must vanish due to the charge conjugation symmetry.

Now the second and third terms in Eq. (63) can be written as

$$
\begin{aligned}
\Pi_{\mu \nu}^{0}(P)= & \sum_{f} \frac{i g^{2}}{2} \int \frac{d^{4} K}{(2 \pi)^{4}} \operatorname{Tr}\left[\gamma_{\mu} S_{0}(K) \gamma_{\nu} S_{0}(Q)\right] \\
= & \sum_{f} \frac{i g^{2}}{2} \int \frac{d^{4} K}{(2 \pi)^{4}}\left[8 K_{\mu} K_{v}-4 K^{2} g_{\mu \nu}\right] \\
& \times \frac{1}{\left(K^{2}-m_{f}^{2}\right)\left(Q^{2}-m_{f}^{2}\right)}
\end{aligned}
$$

$$
\begin{aligned}
\delta \Pi_{\mu \nu}^{a}(P)= & \sum_{f} \frac{i g^{2}}{2} \int \frac{d^{4} K}{(2 \pi)^{4}} \operatorname{Tr}\left[\gamma_{\mu} S_{1}(K) \gamma_{\nu} S_{1}(Q)\right], \\
= & \sum_{f} \frac{i g^{2}}{2}\left(q_{f} B\right)^{2} \\
& \times \int \frac{d^{4} K}{(2 \pi)^{4}} \frac{U_{\mu \nu}}{\left(K^{2}-m_{f}^{2}\right)^{2}\left(Q^{2}-m_{f}^{2}\right)^{2}},
\end{aligned}
$$

where in the numerator we have neglected the mass of the quark and the external momentum $P$ due to HTL approximation. The tensor structure of the self-energy correction in weak field approximation comes out to be

$$
\begin{aligned}
U_{\mu \nu}= & 4(K \cdot u)(Q \cdot u)\left(2 n_{\mu} n_{\nu}+g_{\mu \nu}\right) \\
& +4(K \cdot n)(Q \cdot n)\left(2 u_{\mu} u_{v}-g_{\mu \nu}\right) \\
& -4[(K \cdot u)(Q \cdot n)+(K \cdot n)(Q \cdot u)] \\
& \times\left(u_{\mu} n_{v}+u_{\nu} n_{\mu}\right)+4 m_{f}^{2} g_{\mu \nu} \\
& +8 m_{f}^{2}\left(g_{1 \mu} g_{1 v}+g_{2 \mu} g_{2 v}\right) .
\end{aligned}
$$

The third term in Eq. (63) can be written as

$$
\begin{aligned}
\delta \Pi_{\mu \nu}^{b}(P) & \\
= & \sum_{f} \frac{i g^{2}}{2} \int \frac{d^{4} K}{(2 \pi)^{4}} \operatorname{Tr}\left[\gamma_{\mu} S_{2}(K) \gamma_{\nu} S_{0}(Q)\right] \\
= & \sum_{f} i g^{2}\left(q_{f} B\right)^{2} \int \frac{d^{4} K}{(2 \pi)^{4}} \\
& \times\left[\frac{X_{\mu \nu}}{\left(K^{2}-m_{f}^{2}\right)^{3}\left(Q^{2}-m_{f}^{2}\right)}-\frac{\left(K_{\Perp}^{2}-m_{f}^{2}\right) W_{\mu \nu}}{\left(K^{2}-m_{f}^{2}\right)^{4}\left(Q^{2}-m_{f}^{2}\right)}\right]
\end{aligned}
$$

where

$$
\begin{aligned}
X_{\mu \nu}= & 4\left[(K \cdot u)\left(u_{\mu} Q_{\nu}+u_{\nu} Q_{\mu}\right)\right. \\
- & (K \cdot n)\left(n_{\mu} Q_{\nu}+n_{\nu} Q_{\mu}\right) \\
& \left.+\left\{(K \cdot n)(Q \cdot n)-(K \cdot u)(Q \cdot u)+m_{f}^{2}\right\} g_{\mu \nu}\right],
\end{aligned}
$$

$W_{\mu \nu}=4\left(K_{\mu} Q_{\nu}+Q_{\mu} K_{\mu}\right)-4\left(K \cdot Q-m_{f}^{2}\right) g_{\mu \nu}$.

\subsubsection{Computation of form factors and Debye mass of $\mathcal{O}\left[(e B)^{0}\right]$ term}

In this subsection, we calculate the $\mathcal{O}\left[(e B)^{0}\right]$ terms in the form factors $b, c, d$ in the weak magnetic field limit which are denoted by $b_{0}, c_{0}, d_{0}$, respectively.

The form factor $b_{0}$ in absence of magnetic field can be written from Eq. (25e) as 
$b_{0}\left(p_{0}, p\right)=\frac{1}{\bar{u}^{2}}\left[\Pi_{00}^{g}(P)+\Pi_{00}^{0}(P)\right]$.

where

$$
\begin{aligned}
\Pi_{00}^{0}(P)= & \sum_{f} \frac{i g^{2}}{2} \int \frac{d^{4} K}{(2 \pi)^{4}}\left[8 k_{0}^{2}-4 K^{2}\right] \\
& \times \frac{1}{\left(K^{2}-m_{f}^{2}\right)\left(Q^{2}-m_{f}^{2}\right)} .
\end{aligned}
$$

Using the hard thermal loop (HTL) approximation [38] and performing the frequency sum, one can write

$$
\begin{aligned}
\Pi_{00}^{0}(P)= & -2 g^{2} N_{f} \int \frac{k^{2} d k}{2 \pi^{2}} \frac{d n_{F}(k)}{d k} \\
& \times \int \frac{d \Omega}{4 \pi}\left(1-\frac{p_{0}}{P \cdot \hat{K}}\right),
\end{aligned}
$$

for $m_{f}=0$.

Now the QCD Debye mass in the absence of the magnetic field can directly be obtained using Eq. (25e) as

$$
\begin{aligned}
m_{D}^{2}= & \left.\Pi_{00}^{0}\right|_{\substack{p_{0}=0 \\
\mathbf{p} \rightarrow 0}}=\left.\bar{u}^{2} b_{0}\right|_{\substack{p_{0}=0 \\
\mathbf{p} \rightarrow 0}}=\frac{N_{c} g^{2} T^{2}}{3} \\
& -2 g^{2} \int \frac{k^{2} d k}{2 \pi^{2}} \frac{d n_{F}(k)}{d k}=\frac{g^{2} T^{2}}{3}\left(N_{c}+\frac{N_{f}}{2}\right) .
\end{aligned}
$$

Using Eq. (72) in Eq. (71), we get

$$
\begin{aligned}
\Pi_{00}^{0}(P) & =\frac{N_{f} g^{2} T^{2}}{6} \int \frac{d \Omega}{4 \pi}\left(1-\frac{p_{0}}{p_{0}-\boldsymbol{p} \cdot \hat{\boldsymbol{k}}}\right) \\
& =\frac{N_{f} g^{2} T^{2}}{6}\left(1-\frac{p_{0}}{2 p} \log \frac{p_{0}+p}{p_{0}-p}\right),
\end{aligned}
$$

where we use $p=\sqrt{p_{1}^{2}+p_{3}^{2}}$ as $p$ lies in $x z$ plane as shown Fig. 1. The form factor in Eq. (69) becomes

$b_{0}\left(p_{0}, p\right)=\frac{m_{D}^{2}}{\bar{u}^{2}}\left(1-\frac{p_{0}}{2 p} \log \frac{p_{0}+p}{p_{0}-p}\right)$,

which agrees with the HTL longitudinal form factor $\Pi_{L}\left(p_{0}, p\right)$ [38]. Similarly, we will calculate here the coefficients $c_{0}$ and $d_{0}$ explicitly.

$$
\begin{aligned}
c_{0}\left(p_{0}, p\right)= & R^{\mu v}\left[\Pi_{\mu \nu}^{g}(P)+\Pi_{\mu \nu}^{0}(P)\right] \\
= & \left(\Pi^{g}\right)_{\mu}^{\mu}(P)+\left(\Pi^{0}\right)_{\mu}^{\mu}(P) \\
& +\frac{1}{p_{\perp}^{2}}\left[\left(p_{0}^{2}-p_{\perp}^{2}\right)\left\{\Pi_{00}^{g}(P)+\Pi_{00}^{0}(P)\right\}\right. \\
& +p^{2}\left\{\Pi_{33}^{g}(P)+\Pi_{33}^{0}(P)\right\} \\
& \left.-2 p_{0} p_{3}\left\{\Pi_{03}^{g}(P)+\Pi_{03}^{0}(P)\right\}\right],
\end{aligned}
$$

and

$$
\begin{aligned}
d_{0}\left(p_{0}, p\right)=Q^{\mu \nu}\left[\Pi_{\mu \nu}^{g}(P)+\Pi_{\mu \nu}^{0}(P)\right] & =\frac{p^{2}}{p_{\perp}^{2}}\left[\left\{\Pi_{33}^{g}(P)+\Pi_{33}^{0}(P)\right\}\right. \\
& -\frac{2 p_{0} p_{3}}{p^{2}}\left\{\Pi_{03}^{g}(P)+\Pi_{03}^{0}(P)\right\} \\
& \left.+\frac{p_{0}^{2} p_{3}^{2}}{p^{4}}\left\{\Pi_{00}^{g}(P)+\Pi_{00}^{0}(P)\right\}\right] .
\end{aligned}
$$

Now from Eq. (49), we can write

$$
\begin{aligned}
\Pi_{00}^{g}(P)= & \frac{N_{c} g^{2} T^{2}}{3}\left(1-\frac{p_{0}}{2 p} \log \frac{p_{0}+p}{p_{0}-p}\right), \\
\Pi_{03}^{g}(P)= & \frac{N_{c} g^{2} T^{2}}{3} \frac{p_{0} p_{3}}{p^{2}}\left(1-\frac{p_{0}}{2 p} \log \frac{p_{0}+p}{p_{0}-p}\right), \\
\Pi_{33}^{g}(P)= & \frac{N_{c} g^{2} T^{2}}{3} \frac{3 p_{3}^{2}-p^{2}}{p^{2}} \frac{p_{0}^{2}}{2 p^{2}}\left(1-\frac{p_{0}}{2 p} \log \frac{p_{0}+p}{p_{0}-p}\right) \\
& +\frac{N_{c} g^{2} T^{2}}{3} \frac{p_{3}^{2}-p^{2}}{2 p^{2}} \frac{p_{0}}{2 p} \log \frac{p_{0}+p}{p_{0}-p} .
\end{aligned}
$$

We note that 00 component from the quark contribution $\Pi_{00}^{0}$ is already calculated in Eq. (73) and one needs to calculate the remaining two components of $\Pi_{\mu \nu}^{0}(P)$ which are as follows:

$$
\begin{aligned}
\Pi_{03}^{0}(P) & =\sum_{f} \frac{i g^{2}}{2} \int \frac{d^{4} K}{(2 \pi)^{4}} \frac{8 k_{0} k_{3}}{K^{2} Q^{2}} \\
& =-\frac{N_{f} g^{2} T^{2}}{6} \int \frac{d \Omega}{4 \pi} \frac{p_{0} \hat{k}_{3}}{P \cdot \hat{K}} \\
& =\frac{N_{f} g^{2} T^{2}}{6} \frac{p_{0} p_{3}}{p^{2}}\left(1-\frac{p_{0}}{2 p} \log \frac{p_{0}+p}{p_{0}-p}\right),
\end{aligned}
$$

and

$$
\begin{aligned}
\Pi_{33}^{0}(P)= & \sum_{f} \frac{i g^{2}}{2} \int \frac{d^{4} K}{(2 \pi)^{4}} \frac{8 k_{3}^{2}+4 K^{2}}{\left(K^{2}-m_{f}^{2}\right)\left(Q^{2}-m_{f}^{2}\right)} \\
= & -\frac{N_{f} g^{2} T^{2}}{6} \int \frac{d \Omega}{4 \pi} \frac{p_{0} \hat{k}_{3}^{2}}{P \cdot \hat{K}} \\
= & \frac{N_{f} g^{2} T^{2}}{6} \frac{3 p_{3}^{2}-p^{2}}{p^{2}} \frac{p_{0}^{2}}{2 p^{2}}\left(1-\frac{p_{0}}{2 p} \log \frac{p_{0}+p}{p_{0}-p}\right) \\
& +\frac{N_{f} g^{2} T^{2}}{6} \frac{p_{3}^{2}-p^{2}}{2 p^{2}} \frac{p_{0}}{2 p} \log \frac{p_{0}+p}{p_{0}-p} .
\end{aligned}
$$

Using the results from Eqs. (73), (77)-(81), $c_{0}\left(p_{0}, p\right)$ and $d_{0}\left(p_{0}, p\right)$ become

$$
\begin{aligned}
c_{0}\left(p_{0}, p\right) & =d_{0}\left(p_{0}, p\right) \\
& =\frac{m_{D}^{2}}{2 p^{2}}\left[p_{0}^{2}-\left(p_{0}^{2}-p^{2}\right) \frac{p_{0}}{2 p} \log \frac{p_{0}+p}{p_{0}-p}\right],
\end{aligned}
$$

which agrees with the HTL transverse form factor $\Pi_{T}\left(p_{0}, p\right)$ [38]. 
This implies that the zero magnetic field contribution of the fourth form factor $a$ should vanish. Below we obtain the same from Eqs. (26d) and (64) as,

$$
\begin{aligned}
a_{0}= & \frac{1}{2} N^{\mu \nu}\left[\Pi_{\mu \nu}^{g}+\Pi_{\mu \nu}^{0}\right] \\
= & \frac{1}{2 \sqrt{\bar{u}^{2}} \sqrt{\bar{n}^{2}}}\left[u^{\mu} n^{\nu}+n^{\mu} u^{\nu}-2 \frac{\bar{u} \cdot n}{\bar{u}^{2}} \bar{u}^{\mu} \bar{u}^{\nu}\right] \\
& \times\left[\Pi_{\mu \nu}^{g}+\Pi_{\mu \nu}^{0}\right] \\
= & \frac{1}{2 \sqrt{\bar{u}^{2}} \sqrt{\bar{n}^{2}}}\left[-2 \frac{\bar{u} \cdot n}{\bar{u}^{2}}\left[\Pi_{00}^{g}+\Pi_{00}^{0}\right]+2\left[\Pi_{03}^{g}+\Pi_{03}^{0}\right]\right] \\
= & 0
\end{aligned}
$$

\subsubsection{Computation of form factors and Debye mass of $\mathcal{O}\left[(e B)^{2}\right]$ terms}

In this subsection, we calculate the $\mathcal{O}\left[(e B)^{2}\right]$ coefficients of $b, c, d, a$ which are denoted by $b_{2}, c_{2}, d_{2}, a_{2}$, respectively. The form factor $b_{2}$, i.e., $\mathcal{O}(e B)^{2}$ term of the coefficient $b$, has been computed in Eq. (E13) of appendix E1 as

$$
\begin{aligned}
b_{2}= & \frac{1}{\bar{u}^{2}}\left[\delta \Pi_{00}^{a}(P)+2 \delta \Pi_{00}^{b}(P)\right] \\
= & \frac{\delta m_{D}^{2}}{\bar{u}^{2}}+\sum_{f} \frac{g^{2}\left(q_{f} B\right)^{2}}{\bar{u}^{2} \pi^{2}} \\
& \times\left[\left(g_{k}+\frac{\pi m_{f}-4 T}{32 m_{f}^{2} T}\right)\left(A_{0}-A_{2}\right)\right. \\
& \left.+\left(f_{k}+\frac{8 T-\pi m_{f}}{128 m_{f}^{2} T}\right)\left(\frac{5 A_{0}}{3}-A_{2}\right)\right] .
\end{aligned}
$$

and also the Debye screening mass of $\mathcal{O}(e B)^{2}$ as obtained in Eq. (E8) of appendix E1 as

$$
\begin{aligned}
\delta m_{D}^{2}= & -\sum_{f} \frac{g^{2}}{3 \pi^{2}}\left(q_{f} B\right)^{2}\left[\left(\frac{\partial}{\partial\left(m_{f}^{2}\right)}\right)^{2}+m_{f}^{2}\left(\frac{\partial}{\partial\left(m_{f}^{2}\right)}\right)^{3}\right] \\
& \times m_{f}^{2} \sum_{l=1}^{\infty}(-1)^{l+1}\left[K_{2}\left(\frac{m_{f} l}{T}\right)-K_{0}\left(\frac{m_{f} l}{T}\right)\right] \\
= & \frac{g^{2}}{12 \pi^{2} T^{2}} \sum_{f}\left(q_{f} B\right)^{2} \sum_{l=1}^{\infty}(-1)^{l+1} l^{2} K_{0}\left(\frac{m_{f} l}{T}\right) .
\end{aligned}
$$

We obtain $\mathcal{O}(e B)^{2}$ term of the coefficient $c$ in Eq. (E15) of appendix E2 as

$$
\begin{aligned}
c_{2} & =R^{\mu \nu}\left(\delta \Pi_{\mu \nu}^{a}+2 \delta \Pi_{\mu \nu}^{b}\right) \\
& =-\sum_{f} \frac{4 g^{2}\left(q_{f} B\right)^{2}}{3 \pi^{2}} g_{k}
\end{aligned}
$$

$$
\begin{aligned}
& +\sum_{f} \frac{g^{2}\left(q_{f} B\right)^{2}}{2 \pi^{2}}\left(g_{k}+\frac{\pi m_{f}-4 T}{32 m_{f}^{2} T}\right) \\
& \times\left[-\frac{7}{3} \frac{p_{0}^{2}}{p_{\perp}^{2}}+\left(2+\frac{3}{2} \frac{p_{0}^{2}}{p_{\perp}^{2}}\right) A_{0}\right. \\
& +\left(\frac{3}{2}+\frac{5}{2} \frac{p_{0}^{2}}{p_{\perp}^{2}}+\frac{3}{2} \frac{p_{3}^{2}}{p_{\perp}^{2}}\right) A_{2}-\frac{3 p_{0} p_{3}}{p_{\perp}^{2}} A_{1} \\
& \left.-\frac{5}{2}\left(1-\frac{p_{3}^{2}}{p_{\perp}^{2}}\right) A_{4}-\frac{5 p_{0} p_{3}}{p_{\perp}^{2}} A_{3}\right] .
\end{aligned}
$$

We calculate the $\mathcal{O}(e B)^{2}$ term of the coefficient $d$ in appendix E3 as

$d_{2}=Q^{\mu \nu}\left(\delta \Pi_{\mu \nu}^{a}+2 \delta \Pi_{\mu \nu}^{b}\right)=F_{1}+F_{2}$,

where expressions for $F_{1}$ and $F_{2}$ can be found in Eqs. (E18) and (E19), respectively.

The $\mathcal{O}(e B)^{2}$ term of the coefficient $a$ is calculated in appendix E4 as

$a_{2}=N^{\mu v}\left(\delta \Pi_{\mu \nu}^{a}+2 \delta \Pi_{\mu v}^{b}\right)=G_{1}+G_{2}$,

where $G_{1}$ and $G_{2}$ are given in Eqs. (E21) and (E22) respectively.

\subsubsection{Dispersion}

In weak field approximation the dispersion relation can now be written as

$$
\begin{aligned}
& P^{2}-c=P^{2}-\Pi_{T}-c_{2}=0, \\
& \left(P^{2}-b\right)\left(P^{2}-d\right)-a^{2} \\
& =\left(P^{2}-\Pi_{L}-b_{2}\right)\left(P^{2}-\Pi_{T}-d_{2}\right)-a_{2}^{2} \\
& =\left(P^{2}-\frac{b_{0}+b_{2}+d_{0}+d_{2}+\sqrt{\left(b_{0}+b_{2}-d_{0}-d_{2}\right)^{2}+4 a_{2}^{2}}}{2}\right) \\
& \quad \times\left(P^{2}-\frac{b_{0}+b_{2}+d_{0}+d_{2}-\sqrt{\left(b_{0}+b_{2}-d_{0}-d_{2}\right)^{2}+4 a_{2}^{2}}}{2}\right) \\
& =0
\end{aligned}
$$

which give rise to $c, n^{+}$and $n^{-}$dispersive modes with energies $\omega_{c}, \omega_{n^{+}}$and $\omega_{n^{-}}$respectively.

In this section, we consider that the magnetic field is the smallest scale and calculate all the quantities up to $\mathcal{O}\left[(e B)^{2}\right]$. Within this approximation, Eq. (89b) can be approximated as

$\left(P^{2}-b_{0}-b_{2}\right)\left(P^{2}-d_{0}-d_{2}\right)=0$, 
Fig. 6 Gluon dispersion curves for $\theta_{p}=\pi / 3$ but with varying magnetic field strength $e B=m_{\pi}^{2} / 2, m_{\pi}^{2} / 10$ and $m_{\pi}^{2} /$ $800(\sim 0)$ for $N_{f}=2$
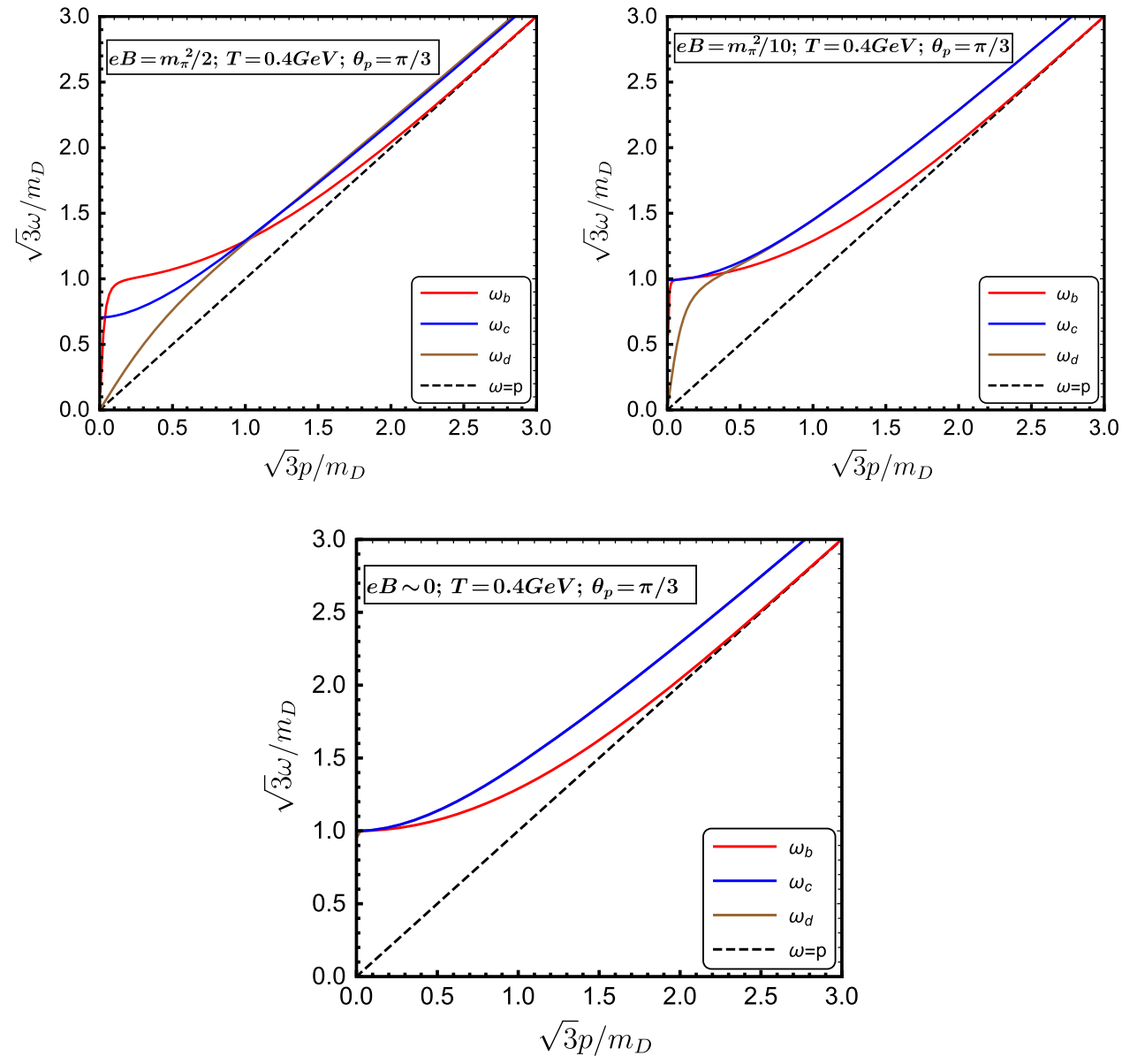

as there is no contribution of $\mathcal{O}\left[(e B)^{2}\right]$ from $a_{2}$ and it only starts contributing $\mathcal{O}\left[(e B)^{4}\right]$ onwards. Thus $a_{2}$ can safely be neglected in the weak field approximation. Now one can write the dispersion relation in weak field approximation as

$P^{2}-b=0$,

$P^{2}-c=0$,

$P^{2}-d=0$,

where the respective dispersive modes are denoted by $b-, c-$, $d$-mode.

We note that the dispersion relations are scaled by plasma frequency of non-magnetized medium, $\omega_{p}=m_{D} / \sqrt{3}$ where $m_{D}^{2}$ is given in Eq. (72). As seen that there are three distinct modes when a gluon propagates in hot magnetized material medium. The magnetized plasmon mode with energy $\omega_{b}$ appears due to the form factor $b$ whereas two transverse modes with energy $\omega_{c}$ and $\omega_{d}$ are, respectively, due to the form factors $c$ and $d$. The presence of magnetic field lifts the degeneracy of the transverse mode found only in a thermal medium.

Now, the dispersion curves for gluon are displayed in Fig. 6 when it propagates at an angle $\theta_{p}=\pi / 3$ with the direction of the magnetic field. We have chosen three different val- ues of magnetic field $|e B|=m_{\pi}^{2} / 2, m_{\pi}^{2} / 10$ and $m_{\pi}^{2} / 800(\sim$ $0) ; m_{\pi}$ is the pion mass. For a given magnetic field strength, say $|e B|=m_{\pi}^{2} / 2$, one finds two modes (viz., $b$ and $d$ mode) with vanishing plasma frequency and one mode (viz., $c$ mode) with finite plasma frequency. The zero plasma frequency for $b$ and $d$ modes could be the artefact of the weak field approximation used in the series expanded version of the Schwinger propagator, i.e. Eq. (61) where the propagator is expanded in a series of $e B$ by considering $e B$ as the lowest scale. This expansion constrains the arbitrariness of the value of $p$ as it is valid only when $p \gtrsim \sqrt{e B}$. Hence in the limit $p \rightarrow 0$ with finite value of $e B$ (however small), as $p$ then becomes the lowest scale and Eq. (61) is not valid. For $d$ mode with a very small magnetic field, the dispersion curve for $d$ at $p=0$ jumps to zero abruptly. This is because, taking $p \rightarrow 0$ limit before taking $e B \rightarrow 0$ again violates the condition $p \gtrsim \sqrt{e B}$ and leaves behind a zero frequency mode. However, the situation is different while taking $e B \rightarrow 0$ limit first though, as in that case considering $e B=0$, one gets back two HTL dispersive modes for gluon propagation. In Fig. 7 we have also displayed the dispersion of gluon when it propagates at an angle $\theta_{p}=\pi / 6$. 
Fig. 7 Gluon dispersion curves for $\theta_{p}=\pi / 6$ but with varying magnetic field strength $e B=m_{\pi}^{2} / 4, m_{\pi}^{2} / 10$ and $m_{\pi}^{2} /$ $800(\sim 0)$ for $N_{f}=2$
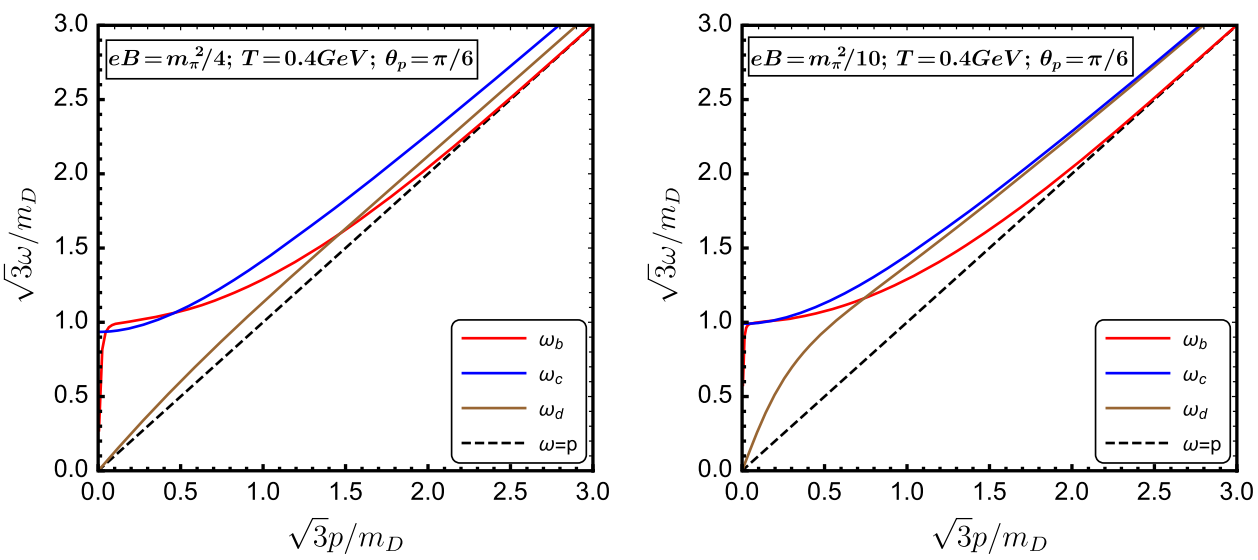

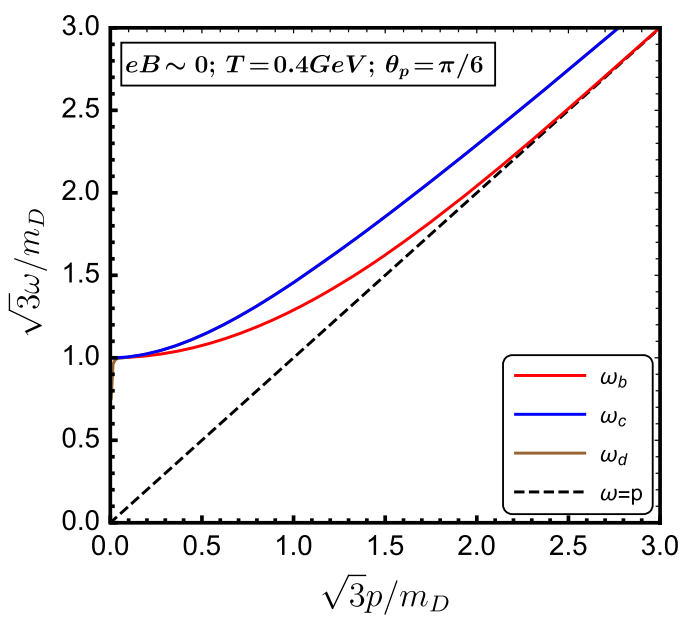

\section{Conclusion}

In this article, we have constructed the general structure of two point functions (self-energy and propagator) of a gauge boson when it travels through a magnetized thermal medium. The Lorentz (boost) invariance is broken due to the presence of heat bath whereas rotational invariance is broken due to the presence of a background magnetic field. Based on gauge invariance and symmetry properties of the gauge boson selfenergy, the general Lorentz structure of gauge boson two point functions is obtained by using four linearly independent basis tensors. We used the effective two point functions to study the dispersion spectra of a gluon in hot magnetized medium. In strong field approximation, one finds three modes which in limiting cases (propagation angle $\pi / 2$ ) merge with the thermal modes. On the other hand in weak field approximation one also finds three distinct modes, viz., one magnetized plasmon, two transverse mode. The calculation for photon can trivially be obtained from this calculation. We further note that the effective propagator obtained here can conveniently be used to study various quantities in QED and QCD plasma. We, finally, note that in a following calculation [36], various thermodynamic quantities are computed using the general structure of the gauge boson here and fermions in Ref. [34] of a magnetized hot QCD plasma.

Acknowledgements Authors gratefully acknowledge the valuable discussion with J. Kapusta on one important technical point in the calculation and a very useful discussion with Palash B Pal and Michael Strickland. The authors would also like to acknowledge the referee for numerous useful comments during the review process. BK would also like to thank Arghya Mukherjee and Avik Banerjee for helpful discussions. AB is supported by the National Post Doctoral Program CAPES (PNPD/CAPES), Govt. of Brazil. NH was funded by the Alexander von Humboldt Foundation, Germany as an Alexander von Humboldt postdoctoral fellow during his stay in Germany. $\mathrm{NH}$ was also supported by the Department of Atomic Energy (DAE), India during his stay in India. BK and MGM were funded by the DAE, India via the project TPAES.

Data Availability Statement This manuscript has no associated data or the data will not be deposited. [Authors' comment: We have not used any associated data in this paper. We used Mathematica to produce the graphs.]

Open Access This article is distributed under the terms of the Creative Commons Attribution 4.0 International License (http://creativecomm ons.org/licenses/by/4.0/), which permits unrestricted use, distribution, and reproduction in any medium, provided you give appropriate credit to the original author(s) and the source, provide a link to the Creative Commons license, and indicate if changes were made. Funded by SCOAP ${ }^{3}$. 


\section{Appendix A: Notation for frequency sum integral}

In imaginary time formalism an integral over loop momentum can be replaced by a frequency sum and an integral over three momentum as

$$
\begin{aligned}
\int \frac{d^{4} K}{(2 \pi)^{4}} \equiv & \sum_{K} \equiv\left(\frac{e^{\gamma_{E}} \Lambda^{2}}{4 \pi}\right)^{\epsilon} i T \sum_{k_{0}=2 n \pi i T} \\
& \times \int \frac{d^{3-2 \epsilon} k}{(2 \pi)^{3-2 \epsilon}}, \\
\int \frac{d^{4} K}{(2 \pi)^{4}} & \equiv \sum_{\{K\}} \equiv\left(\frac{e^{\gamma_{E}} \Lambda^{2}}{4 \pi}\right)^{\epsilon} i T \sum_{k_{0}=(2 n+1) \pi i T} \\
& \times \int \frac{d^{3-2 \epsilon} k}{(2 \pi)^{3-2 \epsilon}},
\end{aligned}
$$

where the loop integral is over Minkowski momentum $K$. Now, the first one is for boson whereas the second one is for fermion. The integral over spatial momentum, in dimensional regularization, is generalized to $d=3-2 \epsilon$ spatial dimensions and $\Lambda$ is an arbitrary momentum scale. The factor $\left(e^{\gamma_{E}} / 4 \pi\right)^{\epsilon}$ is introduced so that, after minimal subtraction of the poles in $\epsilon$ due to ultraviolet divergences, $\Lambda$ coincides with the renormalization scale of the $\overline{\mathrm{MS}}$ renormalization scheme.

\section{Appendix B: Calculation of $\left(\Pi_{\mu}^{\mu}\right)^{s}$ in strong field approx- imation}

Now combining Eqs. (45) and (47) and then contracting with $g^{\mu \nu}$ one can obtain $\left(\Pi_{\mu}^{\mu}\right)^{s}$ as

$$
\begin{aligned}
\left(\Pi_{\mu}^{\mu}\right)^{s}= & -\sum_{f} e^{-p_{\perp}^{2} / 2\left|q_{f} B\right|} \frac{g^{2}\left|q_{f} B\right|}{2 \pi} T \sum_{k_{0}} \\
& \times \int \frac{d k_{3}}{2 \pi} \frac{2 m_{f}^{2}}{\left(K_{\|}^{2}-m_{f}^{2}\right)\left(Q_{\|}^{2}-m_{f}^{2}\right)} .
\end{aligned}
$$

We note that the sum integration after Eq. (B1) is infrared divergent for $m_{f}=0$ in the limit $k_{3} \rightarrow 0$. Below we extract the finite part of it using HTL approximation and the method used in Ref. [54] as

$$
\begin{aligned}
\left(\Pi_{\mu}^{\mu}\right)^{s} \approx & \sum_{f} 2 m_{f}^{2} e^{-p_{\perp}^{2} / 2\left|q_{f} B\right|} \frac{g^{2}\left|q_{f} B\right|}{2 \pi} \int \frac{d k_{3}}{2 \pi} \\
& \times\left[\frac { 1 } { 2 E _ { k _ { 3 } } ^ { 2 } } \left\{\frac{n_{F}\left(E_{k_{3}}\right)}{E_{k_{3}}}+p_{3} \frac{k_{3}}{E_{k_{3}}} \frac{\partial n_{F}\left(E_{k_{3}}\right)}{\partial E_{k_{3}}}\right.\right. \\
& \left.\left.\times \frac{p_{3} k_{3} / E_{k_{3}}}{p_{0}^{2}-p_{3}^{2}\left(k_{3} / E_{k_{3}}\right)^{2}}\right\}\right] \\
\approx & \sum_{f} 2 m_{f}^{2} e^{-p_{\perp}^{2} / 2\left|q_{f} B\right|} \frac{g^{2}\left|q_{f} B\right|}{2 \pi} \int \frac{d k_{3}}{2 \pi}
\end{aligned}
$$

$$
\begin{aligned}
& \times\left[-\frac{\partial}{\partial\left(m_{f}^{2}\right)} \frac{n_{F}\left(E_{k_{3}}\right)}{E_{k_{3}}}\left(1-\frac{p_{0}^{2}}{p_{0}^{2}-p_{3}^{2}}\right)\right. \\
& \left.+\frac{n_{F}\left(E_{k_{3}}\right)}{2 E_{k_{3}}^{3}} \frac{p_{0}^{2}}{p_{0}^{2}-p_{3}^{2}}\right] \\
= & \sum_{f} 2 m_{f}^{2} e^{-p_{\perp}^{2} / 2\left|q_{f} B\right|} \frac{g^{2}\left|q_{f} B\right|}{4 \pi^{2}} \\
& \times\left[-\frac{1}{2 m_{f}^{2}}\left(\frac{p_{3}^{2}}{p_{0}^{2}-p_{3}^{2}}\right)\right. \\
& \left.+\left(\frac{1}{2 m_{f}^{2}}-\frac{\pi}{8 m_{f} T}+\frac{7 \zeta(3)}{8 \pi^{2} T^{2}}\right) \frac{p_{0}^{2}}{p_{0}^{2}-p_{3}^{2}}\right],
\end{aligned}
$$

where we have used Eq. (D10) and the following integrals

$$
\begin{aligned}
& \int_{-\infty}^{\infty} d k_{3} \frac{n_{F}\left(E_{k_{3}}\right)}{E_{k_{3}}}=-\log \frac{m_{f}}{\pi T}-\gamma_{E}, \\
& -\frac{\partial}{\partial\left(m_{f}^{2}\right)} \int_{-\infty}^{\infty} d k_{3} \frac{n_{F}\left(E_{k_{3}}\right)}{E_{k_{3}}}=\frac{1}{2 m_{f}^{2}}, \\
& \int_{-\infty}^{\infty} d k_{3} \frac{n_{F}\left(E_{k_{3}}\right)}{2 E_{k_{3}}^{3}}=\frac{1}{2 m_{f}^{2}}-\frac{\pi}{8 m_{f} T} \\
& +\frac{7 \zeta(3)}{8 \pi^{2} T^{2}} .
\end{aligned}
$$

Now, using Eq. (56) we have

$$
\begin{aligned}
d= & \sum_{f} 2 m_{f}^{2} e^{-p_{\perp}^{2} / 2\left|q_{f} B\right|} \frac{g^{2}\left|q_{f} B\right|}{4 \pi^{2}} \\
& \times\left[-\frac{1}{2 m_{f}^{2}}\left(\frac{p_{3}^{2}}{p_{0}^{2}-p_{3}^{2}}\right)\right. \\
& \left.+\left(\frac{1}{2 m_{f}^{2}}-\frac{\pi}{8 m_{f} T}+\frac{7 \zeta(3)}{8 \pi^{2} T^{2}}\right) \frac{p_{0}^{2}}{p_{0}^{2}-p_{3}^{2}}\right] \\
& +\sum_{f} e^{-p_{\perp}^{2} / 2\left|q_{f} B\right|}\left(\frac{\delta m_{D, f}}{\bar{u}}\right)^{2} \frac{p_{3}^{2}}{p_{0}^{2}-p_{3}^{2}} .
\end{aligned}
$$

\section{Appendix C: Simplification using HTL approximation}

Now, based on HTL approximation we simplify the terms in Eq. (E1) as

$$
\begin{gathered}
\frac{\partial}{\partial\left(m_{f}^{2}\right)} \frac{1}{\left(K^{2}-m_{f}^{2}\right)^{2}\left(Q^{2}-m_{f}^{2}\right)} \\
=\frac{2}{\left(K^{2}-m_{f}^{2}\right)^{3}\left(Q^{2}-m_{f}^{2}\right)} \\
\quad+\frac{1}{\left(K^{2}-m_{f}^{2}\right)^{2}\left(Q^{2}-m_{f}^{2}\right)^{2}} \\
\simeq \frac{3}{\left(K^{2}-m_{f}^{2}\right)^{3}\left(Q^{2}-m_{f}^{2}\right)},
\end{gathered}
$$




$$
\begin{aligned}
& \frac{\partial}{\partial\left(m_{f}^{2}\right)} \frac{1}{\left(K^{2}-m_{f}^{2}\right)^{3}\left(Q^{2}-m_{f}^{2}\right)} \\
& =\frac{3}{\left(K^{2}-m_{f}^{2}\right)^{4}\left(Q^{2}-m_{f}^{2}\right)} \\
& \quad+\frac{1}{\left(K^{2}-m_{f}^{2}\right)^{3}\left(Q^{2}-m_{f}^{2}\right)^{2}} \\
& \simeq \frac{4}{\left(K^{2}-m_{f}^{2}\right)^{4}\left(Q^{2}-m_{f}^{2}\right)}, \\
& \frac{\partial}{\partial\left(m_{f}^{2}\right)} \frac{1}{\left(K^{2}-m_{f}^{2}\right)^{n}\left(Q^{2}-m_{f}^{2}\right)} \\
& \approx \frac{\partial}{\partial\left(k^{2}\right)} \frac{1}{\left(K^{2}-m_{f}^{2}\right)^{n}\left(Q^{2}-m_{f}^{2}\right)} \\
& =\frac{1}{2 k} \frac{\partial}{\partial k} \frac{1}{\left(K^{2}-m_{f}^{2}\right)^{n}\left(Q^{2}-m_{f}^{2}\right)}, \\
& \int \frac{d^{4} K}{(2 \pi)^{4}} \frac{1}{\left(K^{2}-m_{f}^{2}\right)^{2}\left(Q^{2}-m_{f}^{2}\right)} \\
& =-2 \int \frac{d^{4} K}{(2 \pi)^{4}} \frac{k^{2}}{\left(K^{2}-m_{f}^{2}\right)^{3}\left(Q^{2}-m_{f}^{2}\right)} \\
& =\frac{16}{5} \int \frac{d^{4} K}{(2 \pi)^{4}} \frac{k^{4}}{\left(K^{2}-m_{f}^{2}\right)^{4}\left(Q^{2}-m_{f}^{2}\right)}, \\
& \int \frac{d^{4} K}{(2 \pi)^{4}} \frac{1}{\left(K^{2}-m_{f}^{2}\right)^{3}\left(Q^{2}-m_{f}^{2}\right)} \\
& \quad \frac{d^{4} K}{(2 \pi)^{4}} \frac{\left(K^{2}-m_{f}^{2}\right)^{4}\left(Q^{2}-m_{f}^{2}\right)}{} .
\end{aligned}
$$

(C1c)

(C1d)

(C1e)

\section{Appendix D: Frequency sum}

$$
\begin{aligned}
i \int & \frac{d^{4} K}{(2 \pi)^{4}} \frac{1}{\left(K^{2}-m_{f}^{2}\right)\left(Q^{2}-m_{f}^{2}\right)} \\
= & \int \frac{k^{2} d k}{2 \pi^{2}} \int \frac{d \Omega}{4 \pi} \frac{1}{2 E_{k}^{2}} \\
& \times\left[\frac{n_{F}\left(E_{k}\right)}{E_{k}}+\frac{\partial n_{F}\left(E_{k}\right)}{\partial k} \frac{\boldsymbol{p} \cdot \hat{\boldsymbol{k}}}{p_{0}-\boldsymbol{p} \cdot \hat{\boldsymbol{k}} k / E_{k}}\right] \\
= & \int \frac{k^{2} d k}{2 \pi^{2}} \int \frac{d \Omega}{4 \pi} \frac{1}{2 E_{k}^{2}} \\
& \times\left[\frac{n_{F}\left(E_{k}\right)}{E_{k}}+\frac{\partial n_{F}\left(E_{k}\right)}{\partial E_{k}} \frac{\boldsymbol{p} \cdot \hat{\boldsymbol{k}}}{p_{0} E_{k} / k-\boldsymbol{p} \cdot \hat{\boldsymbol{k}}}\right] \\
\approx & \int \frac{k^{2} d k}{2 \pi^{2}} \int \frac{d \Omega}{4 \pi} \frac{1}{2 E_{k}^{2}} \\
& \times\left[\frac{n_{F}\left(E_{k}\right)}{E_{k}}+\frac{\partial n_{F}\left(E_{k}\right)}{\partial E_{k}} \frac{\boldsymbol{p} \cdot \hat{\boldsymbol{k}}}{p_{0}-\boldsymbol{p} \cdot \hat{\boldsymbol{k}}}\right]
\end{aligned}
$$

$$
\begin{aligned}
= & \int \frac{k^{2} d k}{2 \pi^{2}} \int \frac{d \Omega}{4 \pi} \\
& \times\left[\frac{1}{2 E_{k}^{2}} \frac{n_{F}\left(E_{k}\right)}{E_{k}}-\frac{1}{2 E_{k}^{2}} \frac{\partial n_{F}\left(E_{k}\right)}{\partial E_{k}}\right. \\
& \left.+\frac{1}{2 E_{k}^{2}} \frac{\partial n_{F}\left(E_{k}\right)}{\partial E_{k}} \frac{p_{0}}{p_{0}-p \cdot \hat{k}}\right] \\
= & \int \frac{k^{2} d k}{2 \pi^{2}} \int \frac{d \Omega}{4 \pi} \\
& \times\left[-\frac{\partial}{\partial\left(E_{k}^{2}\right)} \frac{n_{F}\left(E_{k}\right)}{E_{k}}+\frac{1}{E_{k}} \frac{\partial n_{F}\left(E_{k}\right)}{\partial\left(E_{k}^{2}\right)} \frac{p_{0}}{P \cdot \hat{K}}\right] \\
= & \int \frac{k^{2} d k}{2 \pi^{2}} \int \frac{d \Omega}{4 \pi} \\
& \times\left[-\frac{\partial}{\partial\left(m_{f}^{2}\right)} \frac{n_{F}\left(E_{k}\right)}{E_{k}}+\frac{1}{E_{k}} \frac{\partial n_{F}\left(E_{k}\right)}{\partial\left(m_{f}^{2}\right)} \frac{p_{0}}{P \cdot \hat{K}}\right] \\
= & \int \frac{k^{2} d k}{2 \pi^{2}} \int \frac{d \Omega}{4 \pi} \\
& \times\left[-\frac{\partial}{\partial\left(m_{f}^{2}\right)} \frac{n_{F}\left(E_{k}\right)}{E_{k}}+\frac{\partial}{\partial\left(m_{f}^{2}\right)} \frac{n_{F}\left(E_{k}\right)}{E_{k}} \frac{p_{0}}{P \cdot \hat{K}}\right. \\
& \left.+\frac{n_{F}\left(E_{k}\right)}{2 E_{k}^{3}} \frac{p_{0}}{P \cdot \hat{K}}\right] \\
& \times\left[1-\frac{p_{0}}{P \cdot \hat{K}}\right]+\int \frac{\left.k^{2}\right)}{2 \pi^{2}} \frac{n_{F}\left(E_{k}\right)}{E_{k}} \int \frac{d \Omega}{4 \pi} \\
\hline \pi^{2} & \frac{d \Omega}{4 \pi} \frac{n_{F}\left(E_{k}\right)}{2 E_{k}^{3}} \frac{p_{0}}{P \cdot \hat{K}}
\end{aligned}
$$

Let us take $m_{f}=y T$ and $k=x T$.

$$
\begin{gathered}
\frac{\partial}{\partial\left(m_{f}^{2}\right)} \int k^{2} d k \frac{n_{F}\left(\sqrt{k^{2}+m_{f}^{2}}\right)}{\sqrt{k^{2}+m_{f}^{2}}} \\
=\frac{\partial}{\partial\left(y^{2}\right)} \int x^{2} d x \frac{n_{F}\left(\sqrt{x^{2}+y^{2}}\right)}{\sqrt{x^{2}+y^{2}}}
\end{gathered}
$$

The integrals can be represented by the well-known functions as,

$$
f_{n+1}(y)=\frac{1}{\Gamma(n+1)} \int_{0}^{\infty} \frac{d x x^{n}}{\sqrt{x^{2}+y^{2}}} n_{F}\left(\sqrt{x^{2}+y^{2}}\right)
$$

which satisfy the following recursion relation,

$$
\frac{\partial f_{n+1}}{\partial y^{2}}=-\frac{f_{n-1}}{2 n}
$$


In the regime of HTL perturbation theory and weak magnetic field, one can use high temperature expansion for $f_{1}$ as,

$$
\begin{aligned}
& f_{1}=-\frac{1}{2} \ln (y / \pi)-\frac{1}{2} \gamma_{E} \\
& \text { So, } \frac{\partial}{\partial\left(m_{f}^{2}\right)} \int \frac{k^{2} d k}{2 \pi^{2}} \frac{n_{F}\left(E_{k}\right)}{E_{k}} \\
& =\frac{1}{8 \pi^{2}}\left[\ln \frac{m_{f}}{\pi T}+\gamma_{E}\right] \\
& \times \int_{0}^{\infty} \frac{d k k^{2}}{2 \pi^{2}} \frac{n_{F}\left(E_{k}\right)}{2 E_{k}^{3}} \int \frac{d \Omega}{4 \pi} \frac{p_{0}}{P \cdot \hat{K}} \\
& =-\frac{1}{8 \pi^{2}}\left[1+\gamma_{E}-\frac{m_{f} \pi}{4 T}+\ln \frac{m_{f}}{\pi T}\right] \\
& \times \int \frac{d \Omega}{4 \pi} \frac{p_{0}}{P \cdot \hat{K}}, \\
& \int \frac{d^{4} K}{(2 \pi)^{4}} \frac{1}{\left(K^{2}-m_{f}^{2}\right)\left(Q^{2}-m_{f}^{2}\right)} \\
& =-\frac{1}{8 \pi^{2}}\left[\ln \frac{m_{f}}{\pi T}+\gamma_{E}\right] \int \frac{d \Omega}{4 \pi}\left[1-\frac{p_{0}}{P \cdot \hat{K}}\right] \\
& -\frac{1}{8 \pi^{2}}\left[1+\gamma_{E}-\frac{m_{f} \pi}{4 T}+\ln \frac{m_{f}}{\pi T}\right] \int \frac{d \Omega}{4 \pi} \frac{p_{0}}{P \cdot \hat{K}} \\
& =-\frac{1}{8 \pi^{2}}\left[\ln \frac{m_{f}}{\pi T}+\gamma_{E}\right] \\
& +\frac{1}{8 \pi^{2}}\left[\frac{m \pi}{4 T}-1\right] \int \frac{d \Omega}{4 \pi} \frac{p_{0}}{P \cdot \hat{K}} \\
& =\frac{1}{8 \pi^{2}} \\
& \times\left[-\ln \frac{m_{f}}{\pi T}-\gamma_{E}+\left(\frac{m_{f} \pi}{4 T}-1\right) \int \frac{d \Omega}{4 \pi} \frac{p_{0}}{P \cdot \hat{K}}\right] .
\end{aligned}
$$$$
i \int \frac{d^{4} K}{(2 \pi)^{4}} \frac{k_{0} k c}{\left(K^{2}-m_{f}^{2}\right)\left(Q^{2}-m_{f}^{2}\right)}
$$$$
=-\int \frac{k^{2} d k}{2 \pi^{2}} k c \frac{\partial n_{F}\left(E_{k}\right)}{\partial\left(m_{f}^{2}\right)} \int \frac{d \Omega}{4 \pi}\left(1-\frac{p_{0}}{P \cdot \hat{K}}\right),
$$$$
i \int \frac{d^{2} K_{॥}}{(2 \pi)^{2}} \frac{1}{\left(K_{\|}^{2}-m_{f}^{2}\right)\left(Q_{\|}^{2}-m_{f}^{2}\right)}
$$$$
=\int_{-\infty}^{\infty} \frac{d k_{3}}{2 \pi}
$$$$
\times\left[\frac{\partial}{\partial\left(m_{f}^{2}\right)} \frac{n_{F}\left(E_{k_{3}}\right)}{E_{k_{3}}} \frac{p_{3}^{2}}{p_{0}^{2}-p_{3}^{2}}+\frac{n_{F}\left(E_{k_{3}}\right)}{2 E_{k_{3}}^{3}} \frac{p_{0}^{2}}{p_{0}^{2}-p_{3}^{2}}\right] \text {, }
$$$$
i \int \frac{d^{2} K_{॥}}{(2 \pi)^{2}} \frac{k_{0} k_{3}}{\left(K_{\|}^{2}-m_{f}^{2}\right)\left(Q_{\|}^{2}-m_{f}^{2}\right)}
$$$$
=\int_{-\infty}^{\infty} \frac{d k_{3}}{2 \pi} \frac{p_{0} p_{3} k_{3}^{2}}{2 E_{k_{3}}^{2}} \frac{\partial n_{F}\left(E_{k_{3}}\right)}{\partial E_{k_{3}}} \frac{1}{p_{0}^{2}-p_{3}^{2} k_{3}^{2} / E_{k_{3}}^{2}} .
$$

(D10)

\section{Appendix E: Calculation of the form factors in weak field approximation}

1. Calculation of the form factor $b_{2}$

$$
\begin{aligned}
b_{2}= & \frac{1}{\bar{u}^{2}}\left[\delta \Pi_{00}^{a}(P)+2 \delta \Pi_{00}^{b}(P)\right] \\
= & \sum_{f} \frac{2 i g^{2}\left(q_{f} B\right)^{2}}{\bar{u}^{2}} \int \frac{d^{4} K}{(2 \pi)^{4}} \\
& \times\left[\frac{K^{2}+\left(1+c^{2}\right) k^{2}+m_{f}^{2}}{\left(K^{2}-m_{f}^{2}\right)^{2}\left(Q^{2}-m_{f}^{2}\right)^{2}}\right. \\
& +\frac{8\left(K^{2}+k^{2}\right)}{\left(K^{2}-m_{f}^{2}\right)^{3}\left(Q^{2}-m_{f}^{2}\right)} \\
& \left.-\frac{8\left(K^{2}+k^{2}\right)\left(K^{2}+\left(1-c^{2}\right) k^{2}-m_{f}^{2}\right)}{\left(K^{2}-m_{f}^{2}\right)^{4}\left(Q^{2}-m_{f}^{2}\right)}\right],
\end{aligned}
$$

where we write $k_{3}$ as $c k$ with $c=\cos \theta$. Using Eqs. (C1a), (C1b), (C1c), and (C1d) obtained in appendix C within HTL approximation, Eq. (E1) becomes

$$
\begin{aligned}
& b_{2}=\sum_{f} \frac{2 i g^{2}\left(q_{f} B\right)^{2}}{\bar{u}^{2}} \\
& \times\left[\left(\frac{\partial}{\partial\left(m_{f}^{2}\right)}+\frac{m_{f}^{2}}{2} \frac{\partial^{2}}{\partial\left(m_{f}^{2}\right)^{2}}\right)\right. \\
& \times \int \frac{d^{4} K}{(2 \pi)^{4}} \frac{\left(1-c^{2}\right)}{\left(K^{2}-m_{f}^{2}\right)\left(Q^{2}-m_{f}^{2}\right)} \\
& +\left(\frac{m_{f}^{2}}{3} \frac{\partial^{2}}{\partial\left(m_{f}^{2}\right)^{2}}\right) \\
& \left.\times \int \frac{d^{4} K}{(2 \pi)^{4}} \frac{1}{\left(K^{2}-m_{f}^{2}\right)\left(Q^{2}-m_{f}^{2}\right)}\right] \\
& =\frac{4 i\left(e^{2} B\right)^{2}}{\bar{u}^{2}} \\
& \times\left[\left(\frac{\partial}{\partial\left(m_{f}^{2}\right)}+\frac{5 m_{f}^{2}}{6} \frac{\partial^{2}}{\partial\left(m_{f}^{2}\right)^{2}}\right)\right. \\
& \times \int \frac{d^{4} K}{(2 \pi)^{4}} \frac{1}{\left(K^{2}-m_{f}^{2}\right)\left(Q^{2}-m_{f}^{2}\right)} \\
& -\left(\frac{\partial}{\partial m_{f}^{2}}+\frac{m_{f}^{2}}{2} \frac{\partial^{2}}{\partial\left(m_{f}^{2}\right)^{2}}\right)
\end{aligned}
$$




$$
\left.\times \int \frac{d^{4} K}{(2 \pi)^{4}} \frac{c^{2}}{\left(K^{2}-m_{f}^{2}\right)\left(Q^{2}-m_{f}^{2}\right)}\right] .
$$

After performing the frequency sum as given in Appendix D, we obtain

$$
\begin{aligned}
& b_{2}=\sum_{f} \frac{g^{2}\left(q_{f} B\right)^{2}}{\bar{u}^{2} \pi^{2}} \\
& \times\left[\left(\frac{\partial^{2}}{\partial\left(m_{f}^{2}\right)^{2}}+\frac{5 m_{f}^{2}}{6} \frac{\partial^{3}}{\partial\left(m_{f}^{2}\right)^{3}}\right)\right. \\
& \times \int k^{2} d k \frac{n_{F}\left(E_{k}\right)}{E_{k}} \int \frac{d \Omega}{4 \pi}\left[\frac{p_{0}}{P \cdot \hat{K}}-1\right] \\
& +\left(\frac{\partial}{\partial\left(m_{f}^{2}\right)}+\frac{5 m_{f}^{2}}{6} \frac{\partial^{2}}{\partial\left(m_{f}^{2}\right)^{2}}\right) \\
& \times \int k^{2} d k \frac{n_{F}\left(E_{k}\right)}{2 E_{k}^{3}} \int \frac{d \Omega}{4 \pi} \frac{p_{0}}{P \cdot \hat{K}} \\
& -\left(\frac{\partial^{2}}{\partial\left(m_{f}^{2}\right)^{2}}+\frac{m_{f}^{2}}{2} \frac{\partial^{3}}{\partial\left(m_{f}^{2}\right)^{3}}\right) \\
& \times \int k^{2} d k \frac{n_{F}\left(E_{k}\right)}{E_{k}} \int \frac{d \Omega}{4 \pi} c^{2}\left[\frac{p_{0}}{P \cdot \hat{K}}-1\right] \\
& -\left(\frac{\partial}{\partial\left(m_{f}^{2}\right)}+\frac{m_{f}^{2}}{2} \frac{\partial^{2}}{\partial\left(m_{f}^{2}\right)^{2}}\right) \\
& \left.\int k^{2} d k \frac{n_{F}\left(E_{k}\right)}{2 E_{k}^{3}} \int \frac{d \Omega}{4 \pi} c^{2} \frac{p_{0}}{P \cdot \hat{K}}\right] \\
& =\frac{3}{2} \frac{\delta m_{D}^{2}}{\bar{u}^{2}} \int \frac{d \Omega}{4 \pi}\left(1-c^{2}\right)\left[1-\frac{p_{0}}{P \cdot \hat{K}}\right] \\
& +\sum_{f} \frac{g^{2}\left(q_{f} B\right)^{2}}{\bar{u}^{2} \pi^{2}} \\
& \times\left\{\left(\frac{\partial}{\partial\left(m_{f}^{2}\right)}+m_{f}^{2} \frac{\partial^{2}}{\partial\left(m_{f}^{2}\right)^{2}}\right)\right. \\
& \times \int k^{2} d k \frac{n_{F}\left(E_{k}\right)}{2 E_{k}^{3}} \int \frac{d \Omega}{4 \pi}\left(1-c^{2}\right) \frac{p_{0}}{P \cdot \hat{K}} \\
& +\frac{m_{f}^{2}}{2} \frac{\partial^{3}}{\partial\left(m_{f}^{2}\right)^{3}} \\
& \times \int k^{2} d k \frac{n_{F}\left(E_{k}\right)}{E_{k}} \int \frac{d \Omega}{4 \pi}\left(\frac{1}{3}-c^{2}\right)
\end{aligned}
$$

$$
\begin{aligned}
& \times\left[1-\frac{p_{0}}{P \cdot \hat{K}}\right]-\frac{m_{f}^{2}}{2} \frac{\partial^{2}}{\partial\left(m_{f}^{2}\right)^{2}} \\
& \left.\times \int k^{2} d k \frac{n_{F}\left(E_{k}\right)}{2 E_{k}^{3}} \int \frac{d \Omega}{4 \pi}\left(\frac{1}{3}-c^{2}\right) \frac{p_{0}}{P \cdot \hat{K}}\right\},
\end{aligned}
$$

where in the second line we have rearranged the terms after using the expression of $\delta m_{D}^{2}$ a obtained following Eq. (25e) as

$$
\begin{aligned}
\delta m_{D}^{2}= & \left.\bar{u}^{2} b_{2}\right|_{p_{0}=0, p \rightarrow 0}=\left[\delta \Pi_{00}^{a}(P)+2 \delta \Pi_{00}^{b}(P)\right]_{p_{0}=0, p \rightarrow 0} \\
= & -\sum_{f} \frac{g^{2}\left(q_{f} B\right)^{2}}{\pi^{2}}\left[\frac{2}{3} \frac{\partial^{2}}{\partial\left(m_{f}^{2}\right)^{2}}+\frac{2}{3} m_{f}^{2} \frac{\partial^{3}}{\partial\left(m_{f}^{2}\right)^{3}}\right] \\
& \times \int k^{2} d k \frac{n_{F}\left(E_{k}\right)}{E_{k}} \\
= & -\sum_{f} \frac{2 g^{2}}{3 \pi^{2}}\left(q_{f} B\right)^{2} \\
& \left.\times \int k^{2} d k \frac{n_{F}^{2}\left(E_{k}\right)}{E_{k}} . m_{f}^{2} \frac{\partial^{3}}{\partial\left(m_{f}^{2}\right)^{3}}\right]
\end{aligned}
$$

There are two types of integrations that appear in Eqs. (E3) and (E4), namely,

$$
\begin{aligned}
& I_{1}=\int k^{2} d k \frac{n_{F}\left(E_{k}\right)}{E_{k}}, \\
& I_{2}=\int k^{2} d k \frac{n_{F}\left(E_{k}\right)}{E_{k}^{3}} .
\end{aligned}
$$

Equation (E5a) can be evaluated in terms of Bessel function as done in Ref. [52] and can be obtained as

$$
\begin{aligned}
I_{1} & =\sum_{l=1}^{\infty}(-1)^{l+1} \int_{0}^{\infty} \frac{k^{2} d k}{\sqrt{k^{2}+m_{f}^{2}}} e^{-\frac{\left(\sqrt{k^{2}+m_{f}^{2}}\right) l}{T}} \\
& =\sum_{l=1}^{\infty}(-1)^{l+1} \frac{m_{f}^{2}}{2}\left[K_{2}\left(\frac{m_{f} l}{T}\right)-K_{0}\left(\frac{m_{f} l}{T}\right)\right] .
\end{aligned}
$$

The second integral in Eq. (E5b) can be evaluated using the procedure described in Ref. [54] and can be obtained at small quark mass as

$I_{2}=-\frac{1}{2}\left[1+\gamma_{E}-\frac{\pi m_{f}}{4 T}+\log \frac{m_{f}}{\pi T}\right]$.

Now, using the Eq. (E6), Eq. (E4) can be written as

$$
\begin{aligned}
\delta m_{D}^{2}= & -\sum_{f} \frac{g^{2}}{3 \pi^{2}}\left(q_{f} B\right)^{2}\left[\frac{\partial^{2}}{\partial\left(m_{f}^{2}\right)^{2}}+m_{f}^{2} \frac{\partial^{3}}{\partial\left(m_{f}^{2}\right)^{3}}\right] \\
& \times m_{f}^{2} \sum_{l=1}^{\infty}(-1)^{l+1}\left[K_{2}\left(\frac{m_{f} l}{T}\right)-K_{0}\left(\frac{m_{f} l}{T}\right)\right]
\end{aligned}
$$




$$
=\sum_{f} \frac{g^{2}}{12 \pi^{2} T^{2}}\left(q_{f} B\right)^{2} \sum_{l=1}^{\infty}(-1)^{l+1} l^{2} K_{0}\left(\frac{m_{f} l}{T}\right),
$$

which agrees with Ref. [52].

Now, we can calculate all the $k$-integrations that appear in Eq. (E3) using the Eqs. (E6) and (E7) as

$$
\begin{aligned}
& \left(\frac{\partial}{\partial\left(m_{f}^{2}\right)}+m_{f}^{2} \frac{\partial^{2}}{\partial\left(m_{f}^{2}\right)^{2}}\right) \int k^{2} d k \frac{n_{F}\left(E_{k}\right)}{2 E_{k}^{3}}=\frac{\pi}{64 T m_{f}}, \\
& \frac{m_{f}^{2}}{2} \frac{\partial^{2}}{\partial\left(m_{f}^{2}\right)^{2}} \int k^{2} d k \frac{n_{F}\left(E_{k}\right)}{2 E_{k}^{3}}=\frac{8 T-\pi m_{f}}{128 T m_{f}^{2}}, \\
& \frac{m_{f}^{2}}{2} \frac{\partial^{3}}{\partial\left(m_{f}^{2}\right)^{3}} \int k^{2} d k \frac{n_{F}\left(E_{k}\right)}{E_{k}}=f_{k} \\
& =-\sum_{l=1}^{\infty}(-1)^{l+1} \frac{l^{2}}{16 T^{2}} K_{2}\left(\frac{m_{f} l}{T}\right), \\
& \frac{\partial^{2}}{\partial\left(m_{f}^{2}\right)^{2}} \int k^{2} d k \frac{n_{F}}{E_{k}}=g_{k} \\
& =\sum_{l=1}^{\infty}(-1)^{l+1} \frac{l}{4 m_{f} T} K_{1}\left(\frac{m_{f} l}{T}\right) .
\end{aligned}
$$

Next, we have to evaluate all the angular integrals of Eq. (E3). The results are given below,

$$
\begin{aligned}
\int \frac{d \Omega}{4 \pi}\left(1-c^{2}\right)\left[1-\frac{p_{0}}{P \cdot \hat{K}}\right] & =\frac{2}{3}-A_{0}+A_{2}, \\
\int \frac{d \Omega}{4 \pi}\left(1-c^{2}\right) \frac{p_{0}}{P \cdot \hat{K}} & =A_{0}-A_{2}, \\
\int \frac{d \Omega}{4 \pi}\left(\frac{1}{3}-c^{2}\right)\left[1-\frac{p_{0}}{P \cdot \hat{K}}\right] & =-\frac{A_{0}}{3}+A_{2}, \\
\int \frac{d \Omega}{4 \pi}\left(\frac{1}{3}-c^{2}\right) \frac{p_{0}}{P \cdot \hat{K}} & =\frac{A_{0}}{3}-A_{2},
\end{aligned}
$$

where $A_{n}$ is defined as

$$
A_{n}=\int \frac{d \Omega}{4 \pi} \frac{p_{0} c^{n}}{P \cdot \hat{K}} \text {. }
$$

$A_{0}$ and $A_{2}$ can now be evaluated as

$$
\begin{aligned}
A_{0}= & \int \frac{d \Omega}{4 \pi} \frac{p_{0}}{P \cdot \hat{K}}=\frac{p_{0}}{2 p} \log \left(\frac{p_{0}+p}{p_{0}-p}\right), \\
A_{2}= & \int \frac{d \Omega}{4 \pi} \frac{c^{2} p_{0}}{P \cdot \hat{K}} \\
= & \frac{p_{0}^{2}}{2 p^{2}}\left(1-\frac{3 p_{3}^{2}}{p^{2}}\right)\left(1-\frac{p_{0}}{2 p} \log \frac{p_{0}+p}{p_{0}-p}\right) \\
& +\frac{1}{2}\left(1-\frac{p_{3}^{2}}{p^{2}}\right) \frac{p_{0}}{2 p} \log \frac{p_{0}+p}{p_{0}-p} .
\end{aligned}
$$

Incorporating all these we finally obtain

$$
\begin{aligned}
b_{2}= & \frac{\delta m_{D}^{2}}{\bar{u}^{2}}+\sum_{f} \frac{g^{2}\left(q_{f} B\right)^{2}}{\bar{u}^{2} \pi^{2}} \\
& \times\left[\left(g_{k}+\frac{\pi m_{f}-4 T}{32 m_{f}^{2} T}\right)\left(A_{0}-A_{2}\right)\right. \\
& \left.+\left(f_{k}+\frac{8 T-\pi m_{f}}{128 m_{f}^{2} T}\right)\left(\frac{5 A_{0}}{3}-A_{2}\right)\right] .
\end{aligned}
$$

\section{Calculation of the form factor $c_{2}$}

In this appendix we calculate the $\mathcal{O}(e B)^{2}$ term of the coefficient $c$ as

$$
\begin{aligned}
c_{2}= & R^{\mu \nu}\left(\delta \Pi_{\mu \nu}^{a}+2 \delta \Pi_{\mu \nu}^{b}\right) \\
= & \sum_{f} \frac{i g^{2}\left(q_{f} B\right)^{2}}{2} \int \frac{d^{4} K}{(2 \pi)^{4}} \\
& \times\left[\frac{4 k_{0}^{2}-4 k_{3}^{2}-4 m_{f}^{2}}{\left(K^{2}-m_{f}^{2}\right)^{2}\left(Q^{2}-m_{f}^{2}\right)^{2}}+\frac{4\left(4 k_{3}^{2}-4 k_{0}^{2}+4 m_{f}^{2}\right)}{\left(K^{2}-m_{f}^{2}\right)^{3}\left(Q^{2}-m_{f}^{2}\right)}\right. \\
& \left.-\frac{4\left(k_{0}^{2}-k_{3}^{2}-m_{f}^{2}\right)\left(8 k_{\perp}^{2}-4 K^{2}+4 m_{f}^{2}+8(\boldsymbol{k} \cdot \boldsymbol{p})_{\perp}^{2} / p_{\perp}^{2}\right.}{\left(K^{2}-m_{f}^{2}\right)^{4}\left(Q^{2}-m_{f}^{2}\right)}\right] \\
= & \sum_{f} 2 i g^{2}\left(q_{f} B\right)^{2} \int \frac{d^{4} K}{(2 \pi)^{4}} \\
& \times\left[\frac{1}{\left(K^{2}-m_{f}^{2}\right)^{2}\left(Q^{2}-m_{f}^{2}\right)}-\frac{k^{2}\left(1-\cos ^{2} \theta\right) \cos ^{2} \phi}{\left(K^{2}-m_{f}^{2}\right)^{3}\left(Q^{2}-m_{f}^{2}\right)}\right. \\
& -\frac{7 k^{2}\left(1-\cos ^{2} \theta\right)\left(1+\cos ^{2} \phi\right)}{\left(K^{2}-m_{f}^{2}\right)^{3}\left(Q^{2}-m_{f}^{2}\right)} \\
& \left.-\frac{8 k^{4} \sin ^{4} \theta\left(1+\cos ^{2} \phi\right)}{\left(K^{2}-m_{f}^{2}\right)^{4}\left(Q^{2}-m_{f}^{2}\right)}\right] .
\end{aligned}
$$

Now, applying HTL approximations, Eq. (E14) can be simplified as

$$
\begin{aligned}
c_{2}= & \sum_{f} 2 i g^{2}\left(q_{f} B\right)^{2} \int \frac{d^{4} K}{(2 \pi)^{4}} \\
& \times\left[\frac{1}{2}+\frac{1}{4}\left(1-\cos ^{2} \theta\right) \cos ^{2} \phi+\frac{7}{4} \sin ^{2} \theta\left(1+\cos ^{2} \phi\right)\right. \\
& \left.-\frac{5}{4} \sin ^{4} \theta\left(1+\cos ^{2} \phi\right)\right] \\
& \times \frac{\partial}{\partial\left(m_{f}^{2}\right)} \frac{1}{\left(K^{2}-m_{f}^{2}\right)\left(Q^{2}-m_{f}^{2}\right)} \\
= & \sum_{f} 2 i g^{2}\left(q_{f} B\right)^{2} \int \frac{d^{4} K}{(2 \pi)^{4}} \\
& \times\left[\frac{1}{2}+2 \sin ^{2} \theta \cos ^{2} \phi+\frac{7}{4} \sin ^{2} \theta\right.
\end{aligned}
$$


658 Page 20 of 23

Eur. Phys. J. C (2019) 79:658

$$
\begin{aligned}
& \left.-\frac{5}{4} \sin ^{4} \theta\left(1+\cos ^{2} \phi\right)\right] \\
& \times \frac{\partial}{\partial\left(m_{f}^{2}\right)} \frac{1}{\left(K^{2}-m_{f}^{2}\right)\left(Q^{2}-m_{f}^{2}\right)} \\
= & -\sum_{f} \frac{4 g^{2}\left(q_{f} B\right)^{2}}{3 \pi^{2}} g_{k} \\
+ & \frac{g^{2}\left(q_{f} B\right)^{2}}{2 \pi^{2}}\left(g_{k}+\frac{\pi m_{f}-4 T}{32 m_{f}^{2} T}\right) \\
\times & {\left[-\frac{7}{3} \frac{p_{0}^{2}}{p_{\perp}^{2}}+\left(2+\frac{3}{2} \frac{p_{0}^{2}}{p_{\perp}^{2}}\right) A_{0}\right.} \\
+ & \left(\frac{3}{2}+\frac{5}{2} \frac{p_{0}^{2}}{p_{\perp}^{2}}+\frac{3}{2} \frac{p_{3}^{2}}{p_{\perp}^{2}}\right) A_{2}-\frac{3 p_{0} p_{3}}{p_{\perp}^{2}} A_{1} \\
& \left.-\frac{5}{2}\left(1-\frac{p_{3}^{2}}{p_{\perp}^{2}}\right) A_{4}-\frac{5 p_{0} p_{3}}{p_{\perp}^{2}} A_{3}\right] .
\end{aligned}
$$

where $g_{k}$ is given in Eq. (E9) and $A_{1}, A_{3}$ and $A_{4}$ are obtained using Eq. (E11) as

$$
\begin{aligned}
A_{1}= & \int \frac{d \Omega}{4 \pi} \frac{c p_{0}}{P \cdot \hat{K}}=-\frac{p_{0} p_{3}}{p^{2}}\left[1-\frac{p_{0}}{2 p} \log \left(\frac{p_{0}+p}{p_{0}-p}\right)\right], \\
A_{3}= & \int \frac{d \Omega}{4 \pi} \frac{c^{3} p_{0}}{P \cdot \hat{K}}=\frac{p_{0}}{2 p} \frac{p_{3}}{p}\left(1-\frac{5}{3} \frac{p_{3}^{2}}{p^{2}}\right) \\
& -\frac{3}{2} \frac{p_{0}}{p} \frac{p_{3}}{p}\left(1-\frac{p_{0}^{2}}{p^{2}}-\frac{p_{3}^{2}}{p^{2}}+\frac{5}{3} \frac{p_{0}^{2}}{p^{2}} \frac{p_{3}^{2}}{p^{2}}\right) \\
& \times\left(1-\frac{p_{0}}{2 p} \log \frac{p_{0}+p}{p_{0}-p}\right), \\
A_{4}= & \int \frac{d \Omega}{4 \pi} \frac{c^{4} p_{0}}{P \cdot \hat{K}} \\
= & \frac{3}{8}\left(1-\frac{p_{3}^{2}}{p^{2}}\right)^{2}-\frac{p_{0}^{2}}{8 p^{2}}\left(1-\frac{5 p_{3}^{2}}{p^{2}}\right)^{2}+\frac{5}{3} \frac{p_{0}^{2}}{p^{2}} \frac{p_{3}^{4}}{p^{4}} \\
& -\frac{3}{8}\left\{\left(1-\frac{p_{0}^{2}}{p^{2}}\right)^{2}-\frac{2 p_{3}^{2}}{p^{2}}\left(1-\frac{3 p_{0}^{2}}{p^{2}}\right)^{2}\right. \\
& \left.+\frac{p_{3}^{4}}{p^{4}}\left(1-\frac{5 p_{0}^{2}}{p^{2}}\right)^{2}+\frac{8 p_{0}^{4}}{p^{4}} \frac{p_{3}^{2}}{p^{2}}\left(1-\frac{5 p_{3}^{2}}{3 p^{2}}\right)\right\} \\
& \left(1-\frac{p_{0}}{2 p} \log \frac{p_{0}+p}{p_{0}-p^{2}}\right) .
\end{aligned}
$$

3. Calculation of the form factor $d_{2}$

In this appendix we compute the form factor $d_{2}$ as

$$
\begin{aligned}
d_{2} & =Q^{\mu v}\left(\delta \Pi_{\mu \nu}^{a}+2 \delta \Pi_{\mu \nu}^{b}\right) \\
& =-\sum_{f} \frac{2 i g\left(q_{f} B\right)^{2} p^{2}}{p_{\perp}^{2}} \int \frac{d^{4} K}{(2 \pi)^{4}}
\end{aligned}
$$

$$
\begin{aligned}
& \times\left[\frac{k_{0}^{2}+k_{3}^{2}-m_{f}^{2}-\frac{4 p_{0} p_{3}}{p^{2}} k_{0} k_{3}+\frac{p_{0}^{2} p_{3}^{2}}{p^{4}}\left(k_{0}^{2}+k_{3}^{2}+m_{f}^{2}\right)}{\left(K^{2}-m_{f}^{2}\right)^{2}\left(Q^{2}-m_{f}^{2}\right)^{2}}\right. \\
+ & 4\left[\frac{k_{3}^{2}+k_{0}^{2}-m_{f}^{2}-\frac{4 p^{0} p^{3}}{p^{2}} k^{0} k^{3}+\frac{p_{0}^{2} p_{3}^{2}}{p^{4}}\left(k_{0}^{2}+k_{3}^{2}+m_{f}^{2}\right)}{\left(K^{2}-m_{f}^{2}\right)^{3}\left(Q^{2}-m_{f}^{2}\right)}\right. \\
& \left.\left.-\frac{\left(k_{0}^{2}-k_{3}^{2}-m_{f}^{2}\right)\left(2 k_{3}^{2}+K^{2}-m_{f}^{2}-\frac{4 p^{0} p^{3}}{p^{2}} k^{0} k^{3}+\frac{p_{0}^{2} p_{3}^{2}}{p^{4}}\left(2 k_{0}^{2}-K^{2}+m_{f}^{2}\right)\right)}{\left(K^{2}-m_{f}^{2}\right)^{4}\left(Q^{2}-m_{f}^{2}\right)}\right)\right] \\
= & \sum_{f} \frac{2 g^{2}\left(q_{f} B\right)^{2} p^{2}}{p_{\perp}^{2}} \int \frac{k^{2} d k}{2 \pi^{2}} \\
& \times\left[\left\{\frac{1}{4}-\left(\frac{3}{2}+\frac{p_{0}^{2} p_{3}^{2}}{p^{4}}\right) c^{2}+\frac{5}{4} c^{4}\right\}\right. \\
& \left.\frac{\partial}{\partial\left(m_{f}^{2}\right)}+m_{f}^{2} \frac{p_{0}^{2} p_{3}^{2}}{2 p^{4}}\left(5-c^{2}\right) \frac{\partial^{2}}{\partial\left(m_{f}^{2}\right)^{2}}\right] \\
& \times\left\{\frac{\partial}{\partial\left(m_{f}^{2}\right)} \frac{n_{F}}{E_{k}}\left(1-\frac{p_{0}}{P \cdot K}\right)-\frac{n_{F}}{2 E_{k}^{3}} \frac{p_{0}}{P \cdot K}\right\} \\
& -\sum_{f} \frac{i g^{2}\left(q_{f} B\right)^{2} p_{0} p_{3}}{3 p_{\perp}^{2}} \int \frac{d^{4} K}{(2 \pi)^{4}} \\
& \times\left[-\frac{\partial^{2}}{\partial\left(m_{f}^{2}\right)^{2}}+k^{2}\left(1-c^{2}\right) \frac{\partial^{3}}{\partial\left(m_{f}^{2}\right)^{3}}\right] \frac{F_{2},}{\left(K^{2}-m_{f}^{2}\right)\left(Q^{2}-m_{f}^{2}\right)}
\end{aligned}
$$

where

$$
\begin{aligned}
& F_{1}=-\sum_{f} \frac{g^{2}\left(q_{f} B\right)^{2} p^{2}}{\pi^{2} p_{\perp}^{2}} \\
& \times\left[-g_{k}\left\{-\frac{p_{0}^{2} p_{3}^{2}}{3 p^{4}}-\frac{A_{0}}{4}+\left(\frac{3}{2}+\frac{p_{0}^{2} p_{3}^{2}}{p^{4}}\right) A_{2}-\frac{5}{4} A_{4}\right\}\right. \\
& +\left(\frac{\pi}{32 m_{f} T}-\frac{1}{8 m_{f}^{2}}\right) \\
& \times\left\{\frac{A_{0}}{4}-\left(\frac{3}{2}+\frac{p_{0}^{2} p_{3}^{2}}{p^{4}}\right) A_{2}+\frac{5}{4} A_{4}\right\} \\
& -f_{k} \frac{p_{0}^{2} p_{3}^{2}}{p^{4}}\left(\frac{14}{3}-5 A_{0}+A_{2}\right) \\
& \left.+\frac{p_{0}^{2} p_{3}^{2}}{p^{4}} \frac{8 T-\pi m_{f}}{128 T m_{f}^{2}}\left(5 A_{0}-A_{2}\right)\right] \text {, } \\
& F_{2}=-\sum_{f} \frac{2 i g^{2}\left(q_{f} B\right)^{2} p^{2}}{p_{\perp}^{2}} \frac{2}{3} \frac{p^{0} p^{3}}{p^{2}} \\
& \times \int \frac{d^{4} K}{(2 \pi)^{4}}\left(-\frac{\partial^{2}}{\partial\left(m_{f}^{2}\right)^{2}}+k^{2}\left(1-c^{2}\right) \frac{\partial^{3}}{\partial\left(m_{f}^{2}\right)^{3}}\right) \\
& \times \frac{k_{0} k c}{\left(K^{2}-m_{f}^{2}\right)\left(Q^{2}-m_{f}^{2}\right)}
\end{aligned}
$$

Springer 


$$
\begin{aligned}
= & -\sum_{f} \frac{g^{2}\left(q_{f} B\right)^{2}}{6 \pi^{2} m_{f} T} \frac{p^{0} p^{3}}{p_{\perp}^{2}} \frac{1}{1+\cosh \frac{m_{f}}{T}} \\
& \times\left(\frac{3 A_{1}}{2}-A_{3}\right) .
\end{aligned}
$$

4. Calculation of the form factor $a_{2}$

$$
\begin{aligned}
& 2 a_{2}=N^{\mu \nu}\left(\delta \Pi_{\mu \nu}^{a}+2 \delta \Pi_{\mu \nu}^{b}\right) \\
& =\sum_{f} \frac{i g^{2}\left(q_{f} B\right)^{2}}{2} \\
& \times \int \frac{d^{4} K}{(2 \pi)^{4}} \frac{N^{\mu \nu} U_{\mu \nu}}{\left(K^{2}-m_{f}^{2}\right)^{2}\left(Q^{2}-m_{f}^{2}\right)^{2}} \\
& +4 i\left(e^{2} B\right)^{2} \int \frac{d^{4} K}{(2 \pi)^{4}} \\
& \times\left[\frac{N^{\mu \nu} X_{\mu \nu}}{\left(K^{2}-m_{f}^{2}\right)^{3}\left(Q^{2}-m_{f}^{2}\right)}\right. \\
& \left.-\frac{\left(k_{0}^{2}-k_{3}^{2}-m_{f}^{2}\right) N^{\mu \nu} W_{\mu \nu}}{\left(K^{2}-m_{f}^{2}\right)^{4}\left(Q^{2}-m_{f}^{2}\right)}\right] \\
& =\sum_{f} \frac{4 i g^{2}\left(q_{f} B\right)^{2}}{\sqrt{\bar{u}^{2}} \sqrt{\bar{n}^{2}}} \int \frac{d^{4} K}{(2 \pi)^{4}} \frac{p_{0} p_{3}}{p^{2}} \\
& \times\left[\left(-1+c^{2}\right) \frac{\partial}{\partial\left(m_{f}^{2}\right)}-\frac{1}{6}\left(5-3 c^{2}\right) m_{f}^{2} \frac{\partial^{2}}{\partial\left(m_{f}^{2}\right)^{2}}\right] \\
& \times \frac{1}{\left(K^{2}-m_{f}^{2}\right)\left(Q^{2}-m_{f}^{2}\right)}+\frac{16 i\left(e^{2} B\right)^{2}}{\sqrt{\bar{u}^{2}} \sqrt{\bar{n}^{2}}} \\
& \times \int \frac{d^{4} K}{(2 \pi)^{4}}\left[\frac{1}{6} \frac{\partial^{2}}{\partial\left(m_{f}^{2}\right)^{2}}-\frac{k^{2}\left(1-c^{2}\right)}{3} \frac{\partial^{3}}{\partial\left(m_{f}^{2}\right)^{3}}\right] \\
& \times \frac{k_{0} k c}{\left(K^{2}-m_{f}^{2}\right)\left(Q^{2}-m_{f}^{2}\right)} \\
& =\sum_{f} \frac{4 g^{2}\left(q_{f} B\right)^{2}}{\sqrt{\bar{u}^{2}} \sqrt{\bar{n}^{2}}} \int \frac{k^{2} d k}{2 \pi^{2}} \int \frac{d \Omega}{4 \pi} \frac{p_{0} p_{3}}{p^{2}} \\
& \times\left[\left(-1+c^{2}\right) \frac{\partial}{\partial\left(m_{f}^{2}\right)}-\frac{1}{6}\left(5-3 c^{2}\right) m_{f}^{2} \frac{\partial^{2}}{\partial\left(m_{f}^{2}\right)^{2}}\right] \\
& \times\left\{-\frac{\partial}{\partial\left(m_{f}^{2}\right)} \frac{n_{F}}{E_{k}}\left(1-\frac{p_{0}}{P \cdot \hat{K}}\right)+\frac{n_{F}}{2 E_{k}^{3}} \frac{p_{0}}{P \cdot \hat{K}}\right\} \\
& -\frac{16\left(e^{2} B\right)^{2}}{\sqrt{\bar{u}^{2}} \sqrt{\bar{n}^{2}}} \int \frac{k^{2} d k}{2 \pi^{2}} \int \frac{d \Omega}{4 \pi} \\
& \times\left[\frac{k c}{6} \frac{\partial^{2}}{\partial\left(m_{f}^{2}\right)^{2}}-\frac{k^{3}\left(c-c^{3}\right)}{3} \frac{\partial^{3}}{\partial\left(m_{f}^{2}\right)^{3}}\right] \\
& \times \frac{\partial n_{F}\left(E_{k}\right)}{\partial\left(m_{f}^{2}\right)}\left(1-\frac{p_{0}}{P \cdot \hat{K}}\right) \\
& =G_{1}+G_{2} \text {, }
\end{aligned}
$$

where

$$
\begin{aligned}
& G_{1}=\sum_{f} \frac{4 g^{2}\left(q_{f} B\right)^{2}}{\sqrt{\bar{u}^{2}} \sqrt{\bar{n}^{2}}} \int \frac{k^{2} d k}{2 \pi^{2}} \int \frac{d \Omega}{4 \pi} \frac{p_{0} p_{3}}{p^{2}} \\
& \times\left[\left(-1+c^{2}\right) \frac{\partial}{\partial\left(m_{f}^{2}\right)}-\frac{1}{6}\left(5-3 c^{2}\right) m_{f}^{2} \frac{\partial^{2}}{\partial\left(m_{f}^{2}\right)^{2}}\right] \\
& \times\left\{-\frac{\partial}{\partial\left(m_{f}^{2}\right)} \frac{n_{F}}{E_{k}}\left(1-\frac{p_{0}}{P \cdot \hat{K}}\right)+\frac{n_{F}}{2 E_{k}^{3}} \frac{p_{0}}{P \cdot \hat{K}}\right\} \\
& =\sum_{f} \frac{4 g^{2}\left(q_{f} B\right)^{2}}{\sqrt{\bar{u}^{2}} \sqrt{\bar{n}^{2}}} \int \frac{k^{2} d k}{2 \pi^{2}} \\
& \times\left[\frac { p _ { 0 } p _ { 3 } } { p ^ { 2 } } \left\{\left(\frac{2}{3}-A_{0}+A_{2}\right) \frac{\partial^{2}}{\partial\left(m_{f}^{2}\right)^{2}}\right.\right. \\
& \left.+\left(\frac{2}{3}-\frac{5 A_{0}}{6}+\frac{A_{2}}{2}\right) m_{f}^{2} \frac{\partial^{3}}{\partial\left(m_{f}^{2}\right)^{3}}\right\} \times \frac{n_{F}}{E_{k}} \\
& +\left\{\left(-A_{0}+A_{2}\right) \frac{\partial}{\partial\left(m_{f}^{2}\right)}\right. \\
& \left.\left.-\frac{1}{6}\left(5 A_{0}-3 A_{2}\right) m_{f}^{2} \frac{\partial^{2}}{\partial\left(m_{f}^{2}\right)^{2}}\right\} \frac{n_{F}}{2 E_{k}^{3}}\right] \\
& =\sum_{f} \frac{4 g^{2}\left(q_{f} B\right)^{2}}{2 \pi^{2} \sqrt{\bar{u}^{2}} \sqrt{\bar{n}^{2}}} \\
& \times\left[\frac{p_{0} p_{3}}{p^{2}}\left\{\left(\frac{2}{3}-A_{0}+A_{2}\right) g_{k}+\left(\frac{4}{3}-\frac{5 A_{0}}{3}+A_{2}\right) f_{k}\right\}\right. \\
& +\left\{\left(-A_{0}+A_{2}\right) \frac{\pi m_{f}-4 T}{32 T m_{f}^{2}}\right. \\
& \left.\left.-\frac{1}{6}\left(5 A_{0}-3 A_{2}\right) \frac{8 T-\pi m_{f}}{64 T m_{f}^{2}}\right\}\right] \text {. } \\
& G_{2}=-\sum_{f} \frac{8 g^{2}\left(q_{f} B\right)^{2}}{\sqrt{\bar{u}^{2}} \sqrt{\bar{n}^{2}}} \int \frac{k^{2} d k}{2 \pi^{2}} \int \frac{d \Omega}{4 \pi} \\
& \times\left[\frac{k c}{6} \frac{\partial^{2}}{\partial\left(m_{f}^{2}\right)^{2}}-\frac{k^{3}\left(c-c^{3}\right)}{3} \frac{\partial^{3}}{\partial\left(m_{f}^{2}\right)^{3}}\right] \\
& \times \frac{\partial n_{F}\left(E_{k}\right)}{\partial\left(m_{f}^{2}\right)}\left(1-\frac{p_{0}}{P \cdot \hat{K}}\right) \\
& =\sum_{f} \frac{g^{2}\left(q_{f} B\right)^{2}}{\sqrt{\bar{u}^{2}} \sqrt{\bar{n}^{2}} 6 \pi^{2} m_{f} T\left(1+\cosh \frac{m_{f}}{T}\right)} \\
& \times\left(-5 A_{1}+4 A_{3}\right) \text {. }
\end{aligned}
$$

\section{References}

1. M. Giovannini, Cosmic microwave background polarization, Faraday rotation, and stochastic gravity-waves backgrounds. Phys. Rev. D 56, 3198 (1997). [arXiv:hep-th/9706201] 
2. M. Giovannini, M.E. Shaposhnikov, Primordial hypermagnetic fields and triangle anomaly. Phys. Rev. D 57, 2186 (1998). arXiv:hep-ph/9710234

3. P.P. Kronberg, Extragalactic magnetic fields. Rep. Prog. Phys. 57, 325 (1994)

4. A. Kosowsky, A. Loeb, Faraday rotation of microwave background polarization by a primordial magnetic field. Astrophys. J. 469, 1 (1996). arXiv:astro-ph/9601055

5. G.D. Fleishman, Q.J. Fu, M. Wang, G.-L. Huang, V.F. Melnikov, Birefringence effect as a tool for astrophysical plasma study. Phys. Rev. Lett. 88, 251101 (2002)

6. A.M. Portis, Electromagnetic Fields: Sources and Media (Wiley, New York, 1978). (see Chap. 12)

7. A. Rai Choudhuri, The Physics of Fluids and Plasmas: An Introduction for Astrophysicists (Cambridge University Press, Cambridge, 1999). (see Chap. 12)

8. S. Ishimaru, Statistical Plasma Physics (Addison-Wesley, Reading, 1992)

9. E.M. Lifshitz, L.P. Pitaevskii, Physical Kinetics (Pergamon, Oxford, 1981)

10. J.F. Nieves, P.B. Pal, $P$ and CP odd terms in the photon selfenergy within a medium. Phys. Rev. D 39, 652 (1989) [Erratum: Phys. Rev. D 40, 2148 (1989)]

11. A.K. Ganguly, S. Konar, P.B. Pal, Faraday effect: a field theoretical point of view'. Phys. Rev. D 60, 105014 (1999). arXiv:hep-ph/9905206

12. J.C. D'Olivo, J.F. Nieves, S. Sahu, Field theory of the photon selfenergy in a medium with a magnetic field and the Faraday effect. Phys. Rev. D 67, 025018 (2003). arXiv:hep-ph/0208146

13. M.H. Thoma, Field theoretic description of ultrarelativistic electron-positron plasmas. Rev. Mod. Phys. 81, 959 (2009). arXiv:0801.0956 [physics.plasm-ph]

14. M.H. Thoma, What can we learn from electromagnetic plasmas about the quark-gluon plasma? J. Phys. A 42, 214004 (2009). https://doi.org/10.1088/1751-8113/42/21/214004. arXiv:0809.1507 [hep-ph]

15. I.A. Shovkovy, Magnetic catalysis: a review. Lect. Notes Phys. 871, 13 (2013). arXiv:1207.5081 [hep-ph]

16. M. D'Elia, Lattice QCD simulations in external background fields. Lect. Notes Phys. 871, 181 (2013). arXiv:1209.0374 [hep-lat]

17. K. Fukushima, Views of the chiral magnetic effect. Lect. Notes Phys. 871, 241 (2013). arXiv:1209.5064 [hep-ph]

18. N. Mueller, J.A. Bonnet, C.S. Fischer, Dynamical quark mass generation in a strong external magnetic field. Phys. Rev. D 89(9), 094023 (2014). arXiv:1401.1647 [hep-ph]

19. V.A. Miransky, I.A. Shovkovy, Quantum field theory in a magnetic field: from quantum chromodynamics to graphene and Dirac semimetals. Phys. Rep. 576, 1 (2015). arXiv:1503.00732 [hep-ph]

20. A. Bzdak, V. Skokov, Anisotropy of photon production: initial eccentricity or magnetic field. Phys. Rev. Lett. 110(19), 192301 (2013). arXiv:1208.5502 [hep-ph]

21. G. Basar, D. Kharzeev, V. Skokov, Conformal anomaly as a source of soft photons in heavy ion collisions. Phys. Rev. Lett. 109, 202303 (2012). arXiv:1206.1334 [hep-ph]

22. K. Fukushima, D.E. Kharzeev, H.J. Warringa, The chiral magnetic effect. Phys. Rev. D 78, 074033 (2008). arXiv:0808.3382 [hep-ph]

23. J. Alexandre, K. Farakos, G. Koutsoumbas, Magnetic catalysis in QED(3) at finite temperature: Beyond the constant mass approximation. Phys. Rev. D 63, 065015 (2001). arXiv:hep-th/0010211

24. V.P. Gusynin, I.A. Shovkovy, Chiral symmetry breaking in QED in a magnetic field at finite temperature. Phys. Rev. D 56, 5251 (1997). arXiv:hep-ph/9704394

25. D.S. Lee, C.N. Leung, Y.J. Ng, Chiral symmetry breaking in a uniform external magnetic field. Phys. Rev. D 55, 6504 (1997). https:// doi.org/10.1103/PhysRevD.55.6504. arXiv:hep-th/9701172
26. D.E. Kharzeev, Topologically induced local $\mathrm{P}$ and $\mathrm{CP}$ violation in QCD x QED. Annals Phys. 325, 205 (2010). arXiv:0911.3715 [hep-ph]

27. G.S. Bali, F. Bruckmann, G. Endrodi, Z. Fodor, S.D. Katz, S. Krieg, A. Schafer, K.K. Szabo, The QCD phase diagram for external magnetic fields. JHEP 1202, 044 (2012). arXiv:1111.4956 [hep-lat]

28. R.L.S. Farias, V.S. Timoteo, S.S. Avancini, M.B. Pinto, G. Krein, Thermo-magnetic effects in quark matter: Nambu-Jona-Lasinio model constrained by lattice QCD. Eur. Phys. J. A 53(5), 101 (2017). arXiv:1603.03847 [hep-ph]

29. A. Ayala, M. Loewe, A.Z. Mizher, R. Zamora, Inverse magnetic catalysis for the chiral transition induced by thermo-magnetic effects on the coupling constant. Phys. Rev. D 90, 036001 (2014)

30. A. Ayala, M. Loewe, R. Zamora, Inverse magnetic catalysis in the linear sigma model with quarks. Phys. Rev. D 91, 016002 (2015). arXiv: 1406.7408 [hep-ph]

31. A. Ayala, C.A. Dominguez, L.A. Hernandez, M. Loewe, R. Zamora, Inverse magnetic catalysis from the properties of the QCD coupling in a magnetic field. Phys. Lett. B 759, 99 (2016). arXiv:1510.09134 [hep-ph]

32. N. Mueller, J.M. Pawlowski, Magnetic catalysis and inverse magnetic catalysis in QCD. Phys. Rev. D 91(11), 116010 (2015). arXiv:1502.08011 [hep-ph]

33. A. Adare et al. [PHENIX Collaboration], Observation of directphoton collective flow in $\sqrt{s_{N N}}=200 \mathrm{GeV} \mathrm{Au+Au} \mathrm{collisions.}$ Phys. Rev. Lett. 109, 122302 (2012). arXiv:1105.4126 [nucl-ex]

34. A. Das, A. Bandyopadhyay, P.K. Roy, M.G. Mustafa, General structure of fermion two-point function and its spectral representation in a hot magnetized medium. Phys. Rev. D 97(3), 034024 (2018). arXiv:1709.08365 [hep-ph]

35. N. Haque, Finite temperature QCD four-point function in the presence of a weak magnetic field within the hard thermal loop approximation. Phys. Rev. D 96(1), 014019 (2017). arXiv:1704.05833 [hep-ph]

36. A. Bandyopadhyay, B. Karmakar, N. Haque, M.G. Mustafa, The pressure of a weakly magnetized hot and dense deconfined QCD matter in one-loop hard-thermal-loop perturbation theory. arXiv:1702.02875 [hep-ph]

37. A. Das, Finite Temperature Field Theory (World Scientific, Singapore, 1997)

38. M. Le Bellac, Thermal Field Theory (Cambridge Monographs on Mathematical Physics) (Cambridge University Press, Cambridge, 1996)

39. J.I. Kapusta, C. Gale, Finite Temperature Field Theory, 2nd edn. (Cambridge University Press, 2006)

40. K. Hattori, D. Satow, Gluon spectrum in a quark-gluon plasma under strong magnetic fields. Phys. Rev. D 97(1), 014023 (2018). arXiv:1704.03191 [hep-ph]

41. A. Ayala, C.A. Dominguez, S. Hernandez-Ortiz, L.A. Hernandez, M. Loewe, D. Manreza Paret, R. Zamora, Thermo-magnetic evolution of the QCD strong coupling. arXiv:1805.08198 [hep-ph]

42. M. Nopoush, Y. Guo, M. Strickland, The static hardloop gluon propagator to all orders in anisotropy. JHEP 1709, 063 (2017). https://doi.org/10.1007/JHEP09(2017)063. arXiv:1706.08091 [hep-ph]

43. P. Romatschke, M. Strickland, Collective modes of an anisotropic quark gluon plasma. Phys. Rev. D 68, 036004 (2003). https://doi. org/10.1103/PhysRevD.68.036004. arXiv:hep-ph/0304092

44. S. Ghosh, A. Mukherjee, P. Roy, S. Sarkar, General structure of neutral $\rho$ meson self energy and its spectral properties in hot and dense magnetized medium. arXiv:1901.02290 [hep-ph]

45. J.O. Andersen, E. Petitgirard, M. Strickland, Phys. Rev. D 70, 045001 (2004). https://doi.org/10.1103/PhysRevD.70.045001. arXiv:hep-ph/0302069 
46. L. McLerran, V. Skokov, Comments about the electromagnetic field in heavy-ion collisions. Nucl. Phys. A 929, 184 (2014). arXiv:1305.0774 [hep-ph]

47. V. Skokov, A.Y. Illarionov, V. Toneev, Estimate of the magnetic field strength in heavy-ion collisions. Int. J. Mod. Phys. A 24, 5925 (2009)

48. R.C. Duncan, C. Thompson, Formation of very strongly magnetized neutron stars-implications for gamma-ray bursts. Astrophys. J. 392, L9 (1992)

49. S. Chakrabarty, D. Bandyopadhyay, S. Pal, Dense nuclear matter in a strong magnetic field. Phys. Rev. Lett. 78, 2898 (1997)

50. D. Bandyopadhyay, S. Chakrabarty, S. Pal, The quantizing magnetic field and quark-hadron phase transition in a neutron star. Phys. Rev. Lett. 79, 2176 (1997)
51. E. Braaten, R.D. Pisarski, Soft amplitudes in hot gauge theories: a general analysis. Nucl. Phys. B 337, 569 (1990)

52. J. Alexandre, Vacuum polarization in thermal QED with an external magnetic field. Phys. Rev. D 63, 073010 (2001). arXiv:hep-th/0009204

53. A. Bandyopadhyay, C.A. Islam, M.G. Mustafa, Electromagnetic spectral properties and Debye screening of a strongly magnetized hot medium. Phys. Rev. D 94(11), 114034 (2016). arXiv:1602.06769 [hep-ph]

54. L. Dolan, R. Jackiw, Symmetry behavior at finite temperature. Phys. Rev. D 9, 3320 (1974) 